\title{
Event-by-Event Fluctuations in Heavy Ion Collisions and the QCD Critical Point
}

\author{
M. Stephanov ${ }^{(a)}$, K. Rajagopal ${ }^{(b)}$ and E. Shuryak ${ }^{(c)}$ \\ (a) Institute for Theoretical Physics, \\ State University of New York, Stony Brook, NY 11794-3840 \\ (b) Center for Theoretical Physics, \\ Massachusetts Institute of Technology, Cambridge, MA 02139 \\ (c) Department of Physics and Astronomy, \\ State University of New York, Stony Brook, NY 11794-3800 \\ March 8 (rev. July 8), 1999; ITP-SB-99-4, MIT-CTP-2834, SUNY-NTG-99-3
}

\begin{abstract}
The event-by-event fluctuations of suitably chosen observables in heavy ion collisions at SPS, RHIC and LHC can tell us about the thermodynamic properties of the hadronic system at freeze-out. By studying these fluctuations as a function of varying control parameters, it is possible to learn much about the phase diagram of QCD. As a timely example, we stress the methods by which present experiments at the CERN SPS can locate the second-order critical endpoint of the first-order transition between quark-gluon plasma and hadron matter. Those event-by-event signatures which are characteristic of freeze-out in the vicinity of the critical point will exhibit nonmonotonic dependence on control parameters. We focus on observables constructed from the multiplicity and transverse momenta of charged pions. We first consider how the event-by-event fluctuations of such observables are affected by Bose-Einstein correlations, by resonances which decay after freeze-out and by fluctuations in the transverse flow velocity. We compare our thermodynamic predictions for such noncritical eventby-event fluctuations with NA49 data, finding broad agreement. We then focus on effects due to thermal contact between the observed pions and a heat bath with a given (possibly singular) specific heat, and due to the direct coupling between the critical fluctuations of the sigma field and the observed pions. We also discuss the effect of the pions produced in the decay of sigma particles just above threshold after freeze-out on the inclusive pion spectrum and on multiplicity fluctuations. We estimate the size of these nonmonotonic effects which appear near the critical point, including restrictions imposed by finite size and finite time, and conclude that they should be easily observable.
\end{abstract}




\section{Introduction and Outline}

The goal of this paper is to motivate a program of heavy ion collision experiments aimed at discovering an important qualitative feature of the QCD phase diagram, namely the critical endpoint at which a line of first order phase transitions separating quark-gluon plasma from hadronic matter comes to an end [1]. The possible existence of such an end point, denoted E, in the temperature $(T)$ vs. baryon chemical potential $(\mu)$ plane has recently been emphasized and its universal critical properties have been described [2, 3]. The point E can be thought of as a descendant of a tricritical point in the phase diagram for 2-flavor QCD with massless

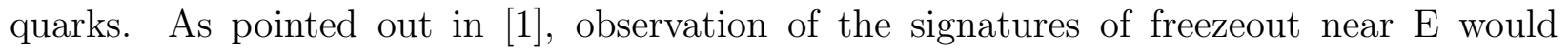
confirm that heavy ion collisions are probing above the chiral transition region in the phase diagram. Furthermore, we would learn much about the qualitative landscape of the QCD phase diagram.

In a previous letter [四], we have laid out the basic ideas for observing the critical endpoint. The signatures proposed in [1] are based on the fact that such a point is a genuine thermodynamic singularity at which susceptibilities diverge and the order parameter fluctuates on long wavelengths. The resulting signatures all share one common property: they are nonmonotonic as a function of an experimentally varied parameter such as the collision energy, centrality, rapidity or ion size. The goal of the present paper is to develop a set of tools which will allow heavy ion collision experiments to discover the critical endpoint through the analysis of the variation of event-by-event fluctuations as control parameters are varied.

Once experimentalists vary a control parameter which causes the freeze-out point in the $(T, \mu)$ plane to move toward, through, and then past the vicinity of the endpoint $\mathrm{E}$, they should see all the signatures we describe first strengthen, reach a maximum, and then decrease, as a nonmonotonic function of the control parameter. It is important to have a control parameter whose variation changes the $\mu$ at which the system crosses the transition region and freezes out. The collision energy is an obvious choice, since it is known experimentally that varying the collision energy has a large effect on $\mu$ at freeze-out. Other possibilities should also be explored."]

An example of nonmonotonic signatures in a different but analogous context is the rise and fall in the number of large fragments as a function of total observed multiplicity in multifragmentation experiments [5] in low energy nuclear collisions. These experiments allow us to confirm the existence and study the properties of another critical point - the end point of the first-order nuclear liquid-gas transition (boiling of the nuclear matter liquid to yield a gas of nucleons) [6, 5]. This point occurs at a temperature of order $10 \mathrm{MeV}$, much lower than the one we are studying [1].

The analogy which is perhaps most familiar is with the phenomenon of critical opalescence observed in most liquids, including water. As the fluid cools down under conditions such that it passes near the end point of the boiling transition, it goes from transparent to opalescent to transparent as the end point is approached and then passed. This nonmonotonic phenomenon

\footnotetext{
${ }^{1}$ If the system crosses the transition region near E, but only freezes out at a much lower temperature, the event-by-event fluctuations will not reflect the thermodynamics near E. In this case, one can push freeze-out to earlier times and thus closer to E by using smaller ions.訨, 此
} 
is due to the scattering of light on critical long wavelength density fluctuations, and thus signals the universal physics unique to the vicinity of the critical point.

The universal property of systems in the vicinity of a second order critical point is the anomalous increase of thermodynamic fluctuations of the order parameter and related observables. Here we consider a specific system, namely the hadronic matter created in a heavy ion collision at the time interactions freeze out. Our generic expectation is that the event-by-event fluctuations of suitable observables increase in the vicinity of a critical endpoint. In this paper we calculate the magnitude of the resulting nontrivial effects, and make predictions which, we hope, will allow experiments to find the endpoint E.

It is clear that before we can achieve this goal we must develop sufficient understanding of non-critical event-by-event fluctuations. Large acceptance detectors, such as NA49 and WA98 at CERN, have made it possible to measure important average quantities in single heavy ion collision events. For example, instead of analyzing the distribution of charged particle transverse momenta obtained by averaging over particles from many events, we can now study the event-by-event variation of the mean transverse momentum of the charged pions in a single event. The event-by-event variation of particle abundance ratios and even of the HBT radii are also becoming available. Although much of this data still has preliminary status, with more statistics and more detailed analysis yet to come, some general features have already been demonstrated. In particular, the event-by-event distributions of these observables are as perfect Gaussians as the data statistics allow, and the fluctuations — the width of the Gaussians — are small. [7]

This is very different from what one observes in $p p$ collisions, in which fluctuations are large. These large non-Gaussian fluctuations clearly reflect non-trivial quantum fluctuations, all the way from the nucleon wave function to that of the secondary hadrons, and are not yet sufficiently well understood. As discussed in [8, 9], thermal equilibration in $A A$ collisions drives the variance of the event-by-event fluctuations down, close to the value determined by the variance of the inclusive one-particle distribution divided by the square root of the multiplicity. In $p p$ physics one can hope to extract quantum mechanical information about the initial state from event-by-event fluctuations of the final state; in heavy ion collisions equilibration renders this an impossible goal. In $A A$ collisions, then, the new goal is to use the much smaller, Gaussian event-by-event fluctuations of the final state to learn about thermodynamic properties at freeze-out.

What can we learn from the magnitude of these small fluctuations and their dependence on the parameters of the collision? Do they contain any more information than the corresponding moments of one-particle inclusive distributions? Some of these questions have been addressed in [10, 11] where it was pointed out that, for example, temperature fluctuations are related to heat capacity via

$$
\frac{\left\langle(\Delta T)^{2}\right\rangle}{T^{2}}=\frac{1}{C_{V}(T)},
$$

and so can tell us about thermodynamic properties of the matter at freeze-out. Similar ideas in [11] relate fluctuations in the occupation of certain momentum bins with $\partial \mu / \partial N$ and the average quantum density in phase space. Furthermore, Mrówczyński has discussed the study of the compressibility of hadronic matter at freeze-out via the event-by-event fluctuations of 
the particle number [12] and Gaździcki [13] and Mrówczyński[14] have considered event-byevent fluctuations of the kaon to pion ratio as measured by NA49[7.

In this paper, we focus on observables constructed from the multiplicity and the momenta of the charged particles in the final state, as measured by NA49. It should be possible to use similar methods to analyze the event-by-event fluctuation of other classes of observables. For example, if it were possible to measure the baryon to pion ratio, analyses analogous to those we discuss would lead to the thermodynamic susceptibility $\partial^{2} \Omega / \partial \mu^{2}$. As the neutrons are not observed, this analysis is not available. However, event-by-event fluctuations of the kaon to pion ratio may yield similar information. Another example is the data obtained by WA98 on the event-by-event fluctuation of the charged particle to photon ratio. 15 They find a Gaussian distribution, and therefore constrain non-equilibrium processes in which long wavelength disorientations of the chiral condensate are excited, as these introduce non-Gaussianity. We leave the extension of the methods of this paper to the study of thermodynamic implications of the NA49 Gaussian distribution of event-by-event $K / \pi$ ratios and of the WA98 Gaussian distribution of event-by-event $\pi^{0} / \pi^{ \pm}$ratios for future work.

Thermodynamic relations like (11) suggest the following strategy. Measure the mean transverse momentum of the charged pions in each event in an ensemble. Since the inclusive average of the transverse momentum of pions from an ensemble of events reflects (although does not equal) the temperature of the ensemble, perhaps one can use $p_{T}$, the mean transverse momentum of the pions in a single event, as a proxy for the temperature of a single event, and so use (1) to obtain $C_{V}$. One of the lessons of the results we present below is that this strategy is too naive. To see a sign of this, consider another fundamental thermodynamic relation, namely that the event-by-event fluctuations of the energy $E$ of a part of a finite system in thermal equilibrium are given by

$$
\left\langle(\Delta E)^{2}\right\rangle=T^{2} C_{V}(T)
$$

For a system in equilibrium, the mean values of $T$ and $E$ are directly related by an equation of state $E(T)$; their fluctuations, however, have quite different behavior as a function of $C_{V}$, and therefore behave differently when $C_{V}$ diverges at a critical point. So, is the $C_{V^{-}}$ dependence of the event-by-event fluctuations of $p_{T}$ like that of $\Delta T$ in (目) or like that of $\Delta E$ in (2)? We will show that $p_{T}$ fluctuations are not like either, although their $C_{V}$-dependence is more similar to that of $\Delta E$, in the sense that the fluctuations of $p_{T}$ grow at the critical point.

Most of our analysis is applied to the fluctuations of the observables characterizing the multiplicity and momenta of the charged pions in the final state of a heavy ion collision. There are several reasons why the pion observables are most sensitive to the critical fluctuations. First, the pions are the most numerous hadrons produced and observed in relativistic heavy ion collisions. A second, very important reason, is that pions couple strongly to the fluctuations of the sigma field (the magnitude of the chiral condensate) which is the order parameter of the phase transition. Indeed, the pions are the quantized oscillations of the phase of the chiral condensate and so it is not surprising that at the critical end point, where

\footnotetext{
${ }^{2}$ We denote the mean transverse momentum of all the pions in a single event by $p_{T}$ rather than $\left\langle p_{T}\right\rangle$ because we choose to reserve $\langle\ldots\rangle$ for averaging over an ensemble of events.
} 
the magnitude of the condensate is fluctuating wildly, signatures are imprinted on the pions. By Section 5, we will have built up the technology needed to analyze these signatures.

Before we outline the structure of the paper, the following comment is in order. We assume throughout that freeze-out occurs from an equilibrated hadronic system. If freezeout occurs "to the left" (lower $\mu$; higher collision energy) of the critical end point E, it occurs after the matter has traversed the crossover region in the phase diagram. If it occurs "to the right" of E, it occurs after the matter has traversed the first order phase transition. This is the situation in which our assumption of freeze-out from an equilibrated system is most open to question. First, one may imagine hadronization directly from the mixed phase, without time for the hadrons to rescatter. Hadronic elastic scattering cross-sections are large enough that this is unlikely. Second, one may worry that the matter is inhomogeneous after the first order transition, and has not had time to re-equilibrate. Fortunately, our assumption is testable. If the matter were inhomogeneous at freeze-out, one can expect non-Gaussian fluctuations in various observables [16] which would be seen in the same experiments that seek the signatures we describe. We focus on the Gaussian thermal fluctuations of an equilibrated system, and study the nonmonotonic changes in these fluctuations associated with moving the freeze-out point toward and then past the critical point, for example from left to right as the collision energy is reduced.

Although our central point is the analysis of the critical fluctuations in the vicinity of the point E, we must first present an extensive analysis of the noncritical fluctuations, which are the background on top of which critical effects must be sought. Thus, this paper is organized as follows: Sections 2 and 3 analyze the background noncritical fluctuations; Section 4 analyzes a particular (negative) contribution to the noncritical fluctuations which disappears near the critical point; Sections 5 and 6 analyze the critical fluctuations themselves.

We begin in Section 2 by discussing the simplest case we can imagine, namely the fluctuations in an ideal Bose gas of pions. This allows us to establish some notation and to explain several conceptual issues. In particular, we explore the relation in this simplest case between the ensemble (i.e., event-by-event) variance and the variance of the inclusive one-particle distribution obtained by averaging over particles from many events. We also point out that the correlation between the multiplicity and an intensive observable, like the mean transverse momentum, only receives contributions from nontrivial effects such as Bose enhancement, energy conservation or interactions. This correlation is in general small, but we see in Sections 4 and 5 that it can increase by a large factor near the critical point. We derive results in Section 2 and throughout which are valid in the thermodynamic limit. In an Appendix, we explain the subtleties of constructing estimators for the relevant quantities using a finite sample of events each with a finite number of pions.

Our goal in Section 3 is the inclusion of various effects neglected in Section 2, except that we continue to assume that freeze-out is not occurring in the vicinity of the critical point. We model the matter in a relativistic heavy ion collision at freeze-out as a resonance gas in thermal equilibrium, and begin by calculating the variance of the event-by-event fluctuations of total multiplicity. Our result suggests that about $75 \%$ of the fluctuations seen in the data are thermodynamic in origin. Our prediction is strongly dependent on the presence of the resonances; had we not included them, our prediction would have been significantly lower, farther below the data. 
Fluctuations in extensive observables like the total multiplicity $N$ are sensitive to nonthermodynamic variation in the initial size of the system which later thermalizes. Sources of such variation include: (i) the distribution of impact parameters; (ii) fluctuation in the initial positions of the nucleons; (iii) quantum fluctuations of the NN cross section [17] described by the wave function of the nucleon, which can be thought of as fluctuations in the effective size of the nucleons at the initial moment of the collision. All these effects lead to fluctuation in the number of spectator nucleons, and thus in the initial size of the interacting system which later thermalizes. We plan to evaluate the size of these contributions to fluctuations in $N$ elsewhere. In this paper, we constrain the magnitude of these nonthermodynamic effects by comparing thermodynamic predictions for the fluctuations in $N$ to the data.

We then turn to a calculation of the variance of the event-by-event fluctuations of the mean transverse momentum, $p_{T}$. This is an intensive variable and should, therefore, be less sensitive to nonthermodynamic variations in the initial size of the system. We calculate numerically the thermodynamic contribution from "direct pions", already present at freezeout, and from the pions generated later by resonance decay. We include Bose effects and the effects of flow and find both to be small. We compare our results to those found by NA49 for central $\mathrm{Pb}-\mathrm{Pb}$ collisions at $160 \mathrm{AGeV}$, and find broad agreement. We do not attempt to include purely experimental effects, such as those due to two-track resolution, and so do not expect precise agreement. Our goal is to compare observed variance with thermodynamic expectations and to see whether they are consistent. Our results support the general idea that the small fluctuations observed in $A A$ collisions, relative to those in $p p$, are consistent with the hypothesis that the matter in the $A A$ collisions achieves approximate local thermal equilibrium in the form of a resonance gas. Once data is available for other collision energies, centralities or ion sizes, the present NA49 data and the calculations of this section will provide an experimental and a theoretical baseline for the study of variation as a function of control parameters.

In Sections 4 and 5, we analyze how the proximity of the critical endpoint to the freezeout point is reflected in the fluctuations. We begin in Section 4 by making the idealization that the pions which one observes are an ideal Bose gas in thermal contact with a heat bath which includes the sigma field. The heat capacity of this heat bath is therefore infinite at the critical point. This treatment neglects the $\sigma \pi \pi$ coupling, which allows the critical fluctuations of the sigma field to influence the pion fluctuations directly, rather than just by thermal contact.

The dominant effects of the critical fluctuations on the pions are the direct effects occurring via the $\sigma \pi \pi$ coupling. The idealization of Section 4 is nevertheless useful, because it allows us to explain and illustrate an important point not made clear in [1] related to the practical application of (1). The fluctuations of the temperature depend on what "mechanical" observable (such as the energy, for example) is measured, and how the measured observable is converted into a temperature. In particular, these fluctuations depend on what part of a system is used as a thermometer. Eq. (1) describes a particular case when the whole system of interest is used as a thermometer. It requires us to use the equation of state, $T(E)$, of the whole system of interest to translate the energy, which is measured in this case, into the temperature [18]. The fluctuations of "mechanical" variables, such as energy, increase at the critical point, as in (2). Because $T(E)$ is singular at the critical point, the fluctuations 
of $T$ decrease, and vanish at the critical point where $C_{V} \rightarrow \infty$. It is a fact that what we measure are the mechanical observables, and since we in general only know $T(E)$ for simple systems we call thermometers, we cannot apply (1) to the complicated system of interest. We illustrate these points by evaluating the fluctuations of several observables in an ideal gas of detected pions (the thermometer) which is in thermal contact with an undetected nonideal, possibly singular, heat bath. The effect we find vanishes at the critical point, where the specific heat of the heat bath diverges due to the fluctuations of the sigmas therein, and so provides a nonmonotonic signature. The effect involves a reduction in the fluctuations of the mean transverse momentum of the pions. What makes it distinctive is that it also involves an anti-correlation between fluctuations of pion occupation numbers with different momenta. We show that this phenomenon follows directly from energy conservation, and conclude that it is much more robust than the idealizations we use to describe it. This signature is present when the system freezes out far from the critical point, and is reduced near the critical point. It should be observable in present data on central $\mathrm{PbPb}$ collisions at $160 \mathrm{AGeV}$, even if freeze-out is not occurring near the critical point in these collisions.

Section 5 describes what we believe to be the dominant event-by-event signatures directly related to the divergent correlation length which characterizes the critical point. We apply much of the technology built up over the preceding sections in Section 5 to study the effect of the interaction of the pions with the almost classical sigma field. We find a large increase in the fluctuations of both the multiplicity and the mean transverse momentum of the pions. This increase would be divergent in the infinite volume limit precisely at the critical point. We apply finite size and finite time scaling to estimate how close the system created in a heavy ion collision can come to the critical singularity, and consequently how large an effect can be seen in the event-by-event fluctuations of the pions. We conclude that the nonmonotonic changes in the variance of the event-by-event fluctuation of the pion multiplicity and momenta which are induced by the universal physics characterizing the critical point can easily be between one and two orders of magnitude greater than the statistical errors in the present data.

Once we have analyzed the effects of the sigma field on the fluctuations of the pions, in Section 6 we ask what becomes of the sigmas themselves. Assuming that freeze-out occurs near the critical point, they are numerous at freeze-out and they can only decay later, once the sigma mass has risen above twice the pion mass. This results in a nonmonotonic signature of the critical point which can be observed even without an event-by-event analysis. We calculate the momentum distribution of these low momentum pions produced in the delayed decays of the sigmas. We close by analyzing the enhanced event-by-event fluctuations of the multiplicity of these low momentum pions.

We end the paper with a summary of the different contributions to the event-by-event fluctuations which we have analyzed, and a more general look to the future. In striving to provide analyses which will assist experimentalists to use the universal properties of the critical point to learn its location, we hope that we have in addition provided a set of tools for event-by-event analyses of heavy ion collisions which will prove useful in the study of the thermodynamics of QCD in a variety of contexts in the future. 


\section{Thermodynamic Fluctuations in an Ideal Bose Gas}

\subsection{The Basics}

We begin by recalling text-book facts about the thermodynamics of an ideal Bose gas which are relevant to our event-by-event analysis. Little in this section is new, but it is nevertheless a very helpful exercise and will allow us to establish some notation. The basic fact is that every quantum state of a system of identical spinless Bose particles is completely characterized by a set of numbers, $n_{p}$ — the occupation numbers for the one-particle states labeled by momenta $p$. All thermodynamic quantities are functions of these numbers and thus all we need to know is the fluctuations of $n_{p}$ from one member of the ensemble (one event) to another. The first step toward a characterization of these fluctuations is the ensemble average of the occupation number for the mode with momentum $p$, namely

$$
\left\langle n_{p}\right\rangle=\frac{1}{e^{\epsilon_{p} / T}-1}
$$

where $\epsilon_{p}=\omega_{p}-\mu$ and, as usual, $\omega_{p}=\sqrt{p^{2}+m^{2}}$. Next, we need the deviation, $\Delta n_{p}=$ $n_{p}-\left\langle n_{p}\right\rangle$, whose mean square average in the ensemble is given by

$$
\left\langle\left(\Delta n_{p}\right)^{2}\right\rangle=T \frac{\partial n_{p}}{\partial \mu}=\frac{e^{\epsilon_{p} / T}}{\left(e^{\epsilon_{p} / T}-1\right)^{2}}=\left\langle n_{p}\right\rangle\left(1+\left\langle n_{p}\right\rangle\right) \equiv v_{p}^{2} .
$$

We have introduced notation $v_{p}^{2}$ for this quantity which will be used frequently below. This expression is "microscopic", in the sense that it is written for a single mode in momentum space. However, it can be derived "macroscopically" as follows. The fluctuations in the total particle number

$$
N=\sum_{p} n_{p}
$$

are given by 18

$$
\left\langle(\Delta N)^{2}\right\rangle=T\left(\frac{\partial N}{\partial \mu}\right)_{T}
$$

Because the fluctuations of different modes are statistically independent, we can elevate this relation to the microscopic form (4), and indeed to

$$
\left\langle\Delta n_{p} \Delta n_{k}\right\rangle=\left\langle\left(\Delta n_{p}\right)^{2}\right\rangle \delta_{p k}=v_{p}^{2} \delta_{p k}
$$

The correlator $\left\langle\Delta n_{p} \Delta n_{k}\right\rangle$ is the central quantity which we will calculate repeatedly throughout this paper, as we proceed beyond the ideal Bose gas.

The correlator in (7) enters in the calculation of the event-by-event mean square deviation of any generic thermodynamic variable of the form

$$
Q=\sum_{p} q_{p} n_{p}
$$


Indeed, since $\Delta Q \equiv Q-\langle Q\rangle=\sum_{p} q_{p} \Delta n_{p}$, we find that

$$
\left\langle(\Delta Q)^{2}\right\rangle=\sum_{p k} q_{p} q_{k}\left\langle\Delta n_{p} \Delta n_{k}\right\rangle=\sum_{p} q_{p}^{2} v_{p}^{2}
$$

The quantity $Q$ could be the total energy

$$
E=\sum_{p} n_{p} \epsilon_{p}, \quad\left\langle(\Delta E)^{2}\right\rangle=\sum_{p} \epsilon_{p}^{2} v_{p}^{2}
$$

or it could be the total transverse momentum, $\sum_{p}\left(p_{T}\right)_{p} n_{p}$; or simply the total particle number

$$
N=\sum_{p} n_{p}, \quad\left\langle(\Delta N)^{2}\right\rangle=\sum_{p} v_{p}^{2}
$$

For future reference we also give here an expression for the heat capacity $C_{V}$ of the Bose gas at constant $V$ and $\mu$ :

$$
C_{V}=T\left(\frac{\partial S}{\partial T}\right)_{\mu}=-T\left(\frac{\partial^{2} \Omega}{\partial T^{2}}\right)_{\mu} .
$$

Using the expression for the thermodynamic potential:

$$
\Omega=T \sum_{p} \ln \left(1-e^{-\epsilon_{p} / T}\right) .
$$

one finds

$$
C_{V}=\frac{1}{T^{2}} \sum_{p} \epsilon_{p}^{2} v_{p}^{2}
$$

Comparing to (10) we find the well-known relation

$$
\left\langle(\Delta E)^{2}\right\rangle=T^{2} C_{V}
$$

which is valid for any system in equilibrium.

\subsection{Energy per Particle: Event-by-Event Average vs. Single- Particle Inclusive Average}

Let us now compute the fluctuation of an intensive observable, such as the mean energy per particle $\epsilon=E / N$, where $E$ and $N$ are extensive, or $p_{T}$, the mean transverse momentum per particle in a single event. Analyzing the member-of-the-ensemble-by-member-of-theensemble fluctuations of the mean energy per particle in a single member of the ensemble is a good warmup. We henceforth begin to refer to members of the ensemble as events. For small fluctuations (and $\Delta E / E \sim N^{-1 / 2} \ll 1$ is small) we can write

$$
\Delta\left(\frac{E}{N}\right) \approx \frac{E}{N}\left(\frac{\Delta E}{E}-\frac{\Delta N}{N}\right)
$$


Now, we square:

$$
\left(\Delta\left(\frac{E}{N}\right)\right)^{2}=\frac{1}{N^{2}}\left((\Delta E)^{2}+\left(\frac{E}{N}\right)^{2}(\Delta N)^{2}-2\left(\frac{E}{N}\right) \Delta E \Delta N\right) .
$$

Then we average. We already know $\left\langle(\Delta E)^{2}\right\rangle$ and $\left\langle(\Delta N)^{2}\right\rangle$, but we also need

$$
\langle\Delta E \Delta N\rangle=\sum_{p} \epsilon_{p} v_{p}^{2}
$$

which is obtained in the same way as before. Putting this all together, we find

$$
\left\langle(\Delta \epsilon)^{2}\right\rangle=\frac{1}{\langle N\rangle^{2}} \sum_{p}\left(\epsilon_{p}-\langle\epsilon\rangle\right)^{2} v_{p}^{2} .
$$

Let us now compare (19) to the variance of the inclusive single particle average energy per particle. To do this, we introduce the notation

$$
\overline{q_{p}} \text { inc } \equiv \sum_{p} q_{p}\left\langle n_{p}\right\rangle / \sum_{p}\left\langle n_{p}\right\rangle=\sum_{p} q_{p}\left\langle n_{p}\right\rangle /\langle N\rangle .
$$

Whereas $\langle\ldots\rangle$ denotes an average over events of some property of a single event, $\ldots$ inc denotes an inclusive average of a property of a single pion over all pions in the ensemble of events, without reference to in which event each pion occurs. It is more convenient for theoretical purposes to work with occupation numbers $n_{p}$, and the inclusive average is then done $n_{p}$ by $n_{p}$ as defined in (20). The subscript ${ }_{p}$ on the left hand side of (20) reminds us that the average is done momentum bin by momentum bin: it is $q_{p}$ which is being averaged, not $q=Q / N$. However, the quantity $\overline{q_{p}}$ inc is $p$-independent. Were we only interested in a quantity like $\langle\epsilon\rangle$, there would be no need to take care with definitions because averaging a single particle quantity pion by pion is the same as first averaging it over an event, and then averaging event-by-event:

$$
\langle\epsilon\rangle=\langle E / N\rangle=\bar{\epsilon}_{p}^{\text {inc }} .
$$

This is not true for fluctuations about the mean, as we see by using our definitions to rewrite (19) as

$$
\left\langle(\Delta \epsilon)^{2}\right\rangle=\frac{1}{\langle N\rangle}{\overline{\left(\epsilon_{p}-\langle\epsilon\rangle\right)^{2}\left(1+\left\langle n_{p}\right\rangle\right)}}^{\text {inc }} .
$$

The same formula holds if $\epsilon=E / N$ is replaced by any quantity of the form $q=Q / N$, for example by the mean transverse momentum per event.

The lesson we learn from (22) is that up to the Bose enhancement factor $\left(1+\left\langle n_{p}\right\rangle\right)$, the ensemble (alias event-by-event) fluctuations of intensive quantities, such as the energy per particle, are indeed given by the variance of the single particle distribution $\overline{\left(\epsilon_{p}-\langle\epsilon\rangle\right)^{2}}$ inc and the central limit theorem which dictates the factor $1 /\langle N\rangle$. We see that the effect of the Bose factor is to increase the variance of the event-by-event distribution relative to that of the inclusive distribution.

When we apply formulae like those we have just derived which are valid in the thermodynamic limit to heavy ion collision data, we will need to construct estimators for the relevant 
quantities using a finite ensemble of events, in which the number of particles in each event is also finite. We describe how this should be done in an Appendix.

Having discussed the fluctuations of extensive and intensive quantities, we end this Section by considering the cross correlation between an intensive observable and the extensive observable $N$. For example, let us calculate $\langle\Delta \epsilon \Delta N\rangle$. Using ingredients we have spelled out above, we find

$$
\langle\Delta \epsilon \Delta N\rangle=\frac{1}{\langle N\rangle} \sum_{p} v_{p}^{2}\left(\epsilon_{p}-\langle\epsilon\rangle\right)=\frac{1}{\langle N\rangle} \sum_{p}\left\langle n_{p}\right\rangle^{2}\left(\epsilon_{p}-\langle\epsilon\rangle\right) .
$$

Note that the terms proportional to $\left\langle n_{p}\right\rangle$ have cancelled, and the remaining term, proportional to $\left\langle n_{p}\right\rangle^{2}$, is obviously due to the Bose effect. This result applies to any such correlation; for example we could have used $p_{T}$ instead of $\epsilon$. The lesson we learn is this: cross correlations between $N$ and intensive observables are generally small, because they receive no contribution if one takes the classical ideal gas limit. Recall that in (21) we find a dominant contribution to the event-by-event variation coming from the variation of the inclusive sin-

gle particle distribution. In (23), this effect cancels and the remaining effects due to Bose enhancement dominate. This means that the nontrivial effects on the pions due to their interactions and due to energy conservation and thermal contact with other degrees of freedom only need to be larger than the effects of Bose enhancement in order to dominate this cross correlation.

\section{Noncritical Thermodynamic Fluctuations in Heavy Ion Collisions}

In this section we proceed to quantitative estimates of the magnitude of noncritical event-byevent fluctuations in heavy ion collisions. As an example of an extensive quantity we use the total charged pion multiplicity of an event; as an example of an intensive quantity we use the mean transverse momentum $p_{T}$ of the charged pions in an event. We compare some of our estimates to preliminary data from the NA49 experiment at CERN SPS on PbPb collisions at $160 \mathrm{AGeV}$, and find broad agreement. In this section, and throughout this paper, we assume thermal equilibrium at freeze-out. In this section, but not throughout this paper, we assume that the system freezes out far from the critical point in the phase diagram, and can be approximated as an ideal resonance gas when it freezes out. The results obtained seem to support the hypotheses that most of the fluctuation observed in the data is indeed thermodynamic in origin and that $\mathrm{PbPb}$ collisions at $160 \mathrm{AGeV}$ do not freeze out near the critical point.

\subsection{Pion Gas at Thermal Freeze-out and Bose Enhancement}

The observed spectrum of pions reflects the distribution of pion momenta at the time of thermal freeze-out, namely the time at which the interaction rates fall behind the expansion rate. After this time, one can approximately neglect energy/momentum exchange interactions and consider the momenta of particles as frozen. Freeze-out is by definition the time at which the 
system ceases to be in thermal equilibrium. However, if the system has thermalized before it freezes out, then even after freeze-out one has a thermal distribution of pion momenta, [3 approximately with a single temperature over the whole system. This standard idealization at this point seems sufficient to describe the data. (Particles which interact more weakly than pions freeze out earlier, at a higher temperature. We leave such particles, together with details of the dynamics of the freeze-out of the pions, to future work.)

We start with the simplest model for the pions at freeze-out - the ideal Bose gas. This allows us to use the results of the previous section. Later in this section, we add pions produced by the decay of resonances as well. The isospin degeneracy of the pions requires a small modification to the formulae of the previous section. Since only the momenta of charged pions are observed, we must only count $\pi^{+}$and $\pi^{-}$. Because $\pi^{+}$and $\pi^{-}$are distinct, the Bose enhancement factor is reduced from $1+n_{p}$ to $1+\left(n_{p} / 2\right)$, where $n_{p}$ counts the total number of charged pions. This is the consequence of the fact that only identical pions can interfere. f $^{2}$

We begin by showing that the effect of the Bose enhancement is very sensitive to a nonzero pion chemical potential $\mu_{\pi}$ (not to be confused with the baryon number chemical potential $\mu$ ). Let us first remind the reader why a nonzero $\mu_{\pi}$ may be needed. The pion chemical potential is not a thermodynamic conjugate to any fundamentally conserved quantity, and is the same for pions of all charges. It is supposed to represent the over-population of pion phase space. It allows for the possibility that even though the momenta of the pions are in equilibrium at freeze-out, their number density is not. This arises because all reactions which can change the number of particles, and thus keep this quantity in equilibrium, have small cross sections at the relevant low energies. In contrast, elastic re-scattering is strongly enhanced by resonances (such as $\Delta, N^{*}$ for $\pi N, \sigma, \rho$ for $\pi \pi$ etc). As a result, thermal equilibrium of momenta is maintained to a lower freeze-out temperature, whereas chemical freeze-out (below which particle numbers do not change) occurs somewhat earlier. There is therefore a window of time between chemical and thermal freeze-out during which the system evolves with fixed pion number; during this time a pion chemical potential naturally develops. At chemical freeze-out, $\mu_{\pi}=0$. As the temperature then continues to drop while the pion number remains fixed, $\mu_{\pi}$ increases. For an overview of pion kinetics and references see [19]. Practical calculations of the magnitude of the effect for heavy ion collisions at CERN SPS can be found in [20]. The conclusion inferred from this analysis is that the pions in central $\mathrm{PbPb}$ collisions at SPS energies freeze out at a temperature $T_{\mathrm{f}} \approx 120 \mathrm{MeV}$ with $\mu_{\pi} \approx 60 \mathrm{MeV}$.

Now we return to the calculation of the Bose enhancement of fluctuations of some generic single-particle intensive observable $q=Q / N$. If we use the notation $v_{\text {ebe }}$ for the event-byevent variance and $v_{\text {inc }}$ for the variance of the inclusive distribution:

$$
v_{\text {ebe }}^{2}(q)=\left\langle(\Delta q)^{2}\right\rangle \quad \text { and } \quad v_{\text {inc }}^{2}(q)={\overline{\left(q_{p}-\langle q\rangle\right)^{2}}}^{\text {inc }}
$$

\footnotetext{
${ }^{3} \mathrm{As}$ is very accurately the case for the cosmic microwave background radiation, ten billion years after its freeze-out.

${ }^{4}$ It is easy to see that $\left\langle\Delta n_{p}^{i} \Delta n_{k}^{j}\right\rangle=\left\langle n_{p}^{i}\right\rangle\left(1+\left\langle n_{p}^{i}\right\rangle\right) \delta^{i j} \delta_{p k}$, where $i, j=+,-$. On the other hand, from $n_{p}=\sum_{i} n_{p}^{i}$ it follows that: $\left\langle n_{p}^{i}\right\rangle=\left\langle n_{p}\right\rangle / 2$, and $\left\langle\Delta n_{p} \Delta n_{k}\right\rangle=\left\langle n_{p}\right\rangle\left(1+\left\langle n_{p}\right\rangle / 2\right) \delta_{p k}$.
} 
and define the ratio

$$
F \equiv \frac{\langle N\rangle v_{\mathrm{ebe}}^{2}(q)}{v_{\text {inc }}^{2}(q)}
$$

then we can write the result (22) for the ideal Bose gas as

$$
F=F_{B} \equiv 1+\frac{1}{2} \frac{\int d^{3} p\left(q_{p}-\langle q\rangle\right)^{2}\left\langle n_{p}\right\rangle^{2}}{\int d^{3} p\left(q_{p}-\langle q\rangle\right)^{2}\left\langle n_{p}\right\rangle} .
$$

The factor of $1 / 2$ appears because, as discussed above, there are two charged pions. As we consider effects not present in an ideal gas, we will find that the ratio $F$ is not given simply by the Bose enhancement factor $F_{B}$. It is a product of $F_{B}$ and other factors which we estimate later in this section and in subsequent sections.

The dependence of $F_{B}$ on $\mu_{\pi}$ is shown in Fig. 1 for $q=p_{T}=\sqrt{p_{x}^{2}+p_{y}^{2}}$. (Note that $v_{\text {inc }}^{2}$ does depend on the pion chemical potential as well.) We have also shown $F_{B}$ for pions with $p_{T}<300 \mathrm{MeV}$ to demonstrate that restricting the acceptance to low-energy pions results in a larger Bose enhancement effect. It is worth noting that for more central collisions the thermal freeze-out temperature, $T_{\mathrm{f}}$, is lower because the system is larger and freezes out later [ [1]. Therefore, $\mu_{\pi}$ should be somewhat larger and the Bose enhancement effect should somewhat increase event-by-event fluctuations for more central collisions. To conclude, the

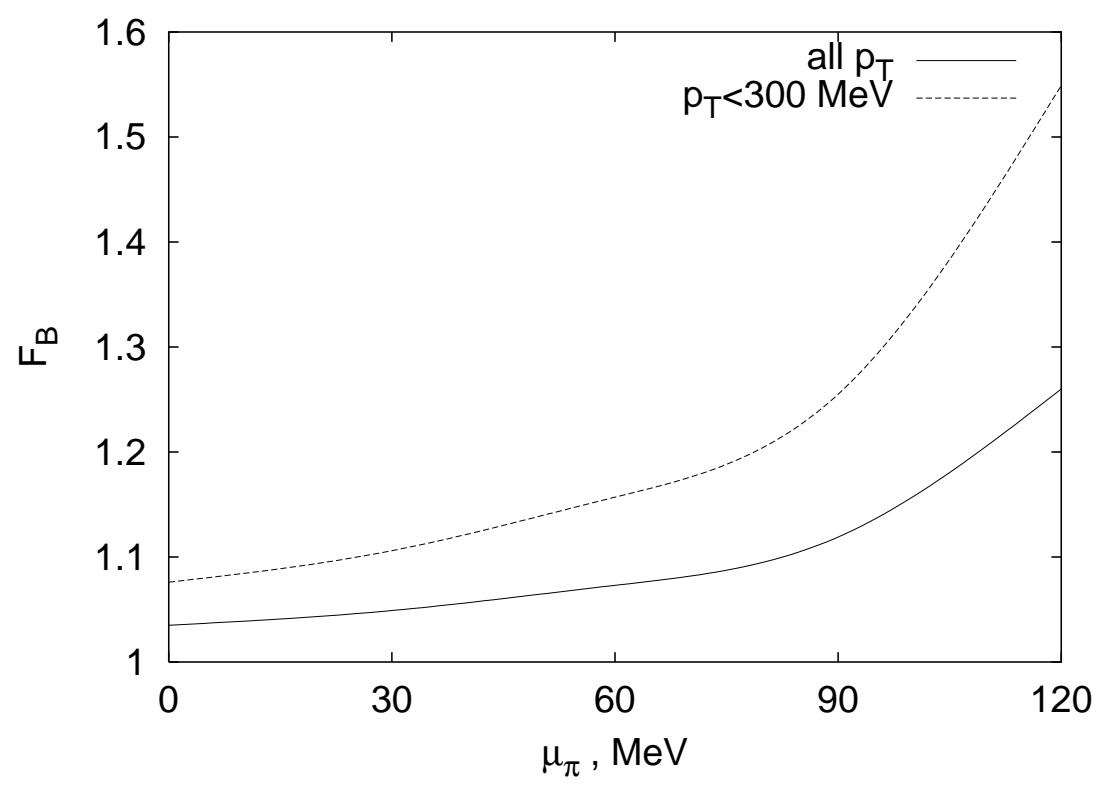

Figure 1: Bose enhancement factor $F_{B}=\langle N\rangle v_{\text {ebe }}^{2}\left(p_{T}\right) / v_{\text {inc }}^{2}\left(p_{T}\right)$ describing the contribution of the Bose effects to the fluctuations of the mean transverse momentum in an ideal Bose gas of pions. $F_{B}$ is plotted as a function of the pion chemical potential $\mu_{\pi}$, in $\mathrm{MeV}$. The dashed line shows the Bose enhancement factor if only pions with low momentum $p_{T}<300 \mathrm{MeV}$ are included.

Bose enhancement effect is sensitive to $\mu_{\pi}$, and leads to an increase in $v_{\text {ebe }}$ by a factor of $\sqrt{F_{B}}$, which typically results in an increase of the order of a few percent. 
The effects of Bose enhancement on the variance of the fluctuations of $p_{T}$ in an ideal Bose gas have been considered previously [21, 7]: our results are in quantitative agreement with theirs. These authors use the quantity

$$
\Phi_{p_{T}}=\langle N\rangle^{1 / 2} v_{\text {ebe }}\left(p_{T}\right)-v_{\text {inc }}\left(p_{T}\right)
$$

introduced in Ref. [8] as a measure of the Bose enhancement effect. As we discuss in the Appendix and below, when $\langle N\rangle$ is finite one must take care in defining $\Phi_{p_{T}}$ to use an appropriate estimator for $v_{\text {ebe }}\left(p_{T}\right)$. To compare our results with theirs, note that what we describe as $\sqrt{F_{B}}=1.01$ corresponds to $\Phi_{p_{T}}=(0.01) v_{\text {inc }}\left(p_{T}\right)$. This already hints at what we will see below, namely that whereas $\Phi_{p_{T}}$ depends on the flow velocity through $v_{\text {inc }}\left(p_{T}\right)$, ratios like $F_{B}$ are much less sensitive to the effects of flow and are therefore more easy to calculate.

\subsection{Contribution of Resonances}

The hadronic matter produced in a heavy ion collision is not simply an ideal gas of pions. A number of approaches to heavy ion collisions have successfully treated the matter at freezeout as a resonance gas in thermal equilibrium. Our analysis of the fluctuations observed in present data lends support to this idea.

Even the global properties of hadronic matter are strongly affected by resonances. Although at temperatures of interest $\left(T<T_{c}\right)$ the Boltzmann factor $\exp (-M / T)$ for each resonance is small, it is partially compensated by the pre-exponential factor due to the large number of states involved. One may recall here the Hagedorn conjecture, that at a certain temperature the contribution to the energy density due to the resonances would diverge because of the exponential growth of the density of states. Although this does not happen in practice, because the chiral phase transition occurs at a much lower temperature than any putative Hagedorn transition, one nevertheless finds [22] that when relevant resonances are included, the energy density and pressure increase rapidly with $T$, and can be fitted by a power law

$$
\varepsilon(T) \sim P(T) \sim T^{\kappa}
$$

with the power $\kappa \approx 6$ at zero baryon density. . One also finds that the heat capacity, normalized to the number of pions (which means that we have "decayed" all resonances, counting each $\rho$ meson as 2 pions, each $\omega$ as 3 , etc.) is

$$
\left(C_{V} / N_{\pi}\right)_{\text {resonance gas }} \approx 23,
$$

at $T_{f}=120 \mathrm{MeV}$, while for the ideal Bose gas of pions one has only:

$$
\left(C_{V} / N_{\pi}\right)_{\text {pions }} \approx 14
$$

at the same temperature. This general observation [11] already suggests that the resonances may affect the fluctuations considerably.

\footnotetext{
${ }^{5}$ For nonzero baryon density this effective power is even larger, but we will ignore this since it is only important at much higher baryon densities (and lower collision energies) than achieved at the CERN SPS.
} 
The resonances play another role in the problem. Those which are present at freeze-out decay after freeze-out, and by definition this means that the pions they produce cannot rescatter. The pions observed in the data are therefore a sum of (i) "direct pions" which were pions at freeze-out and (ii) pions produced from the decay of resonances after freezeout. Note that the direct pions also originate from resonances, in the sense that most of the low energy rescattering which occurs before freeze-out occur via resonances. As we have discussed above, inelastic reactions which change the number of participants freeze-out earlier than elastic scattering. What this means is that the multiplicities of the pions and resonances, although thermal, should be fixed not at the thermal freeze-out $\left(T_{\mathrm{f}} \sim 120 \mathrm{MeV}\right.$ in $\mathrm{PbPb}$ ) but earlier, at chemical freeze-out. Fits of SPS data on ratios of particle yields to thermal models yield $T_{\mathrm{ch}} \sim 160-170 \mathrm{MeV} 23$.

In the remainder of this subsection, we investigate three effects of the resonances on the event-by-event fluctuations of the extensive observable $N$, the number of charged pions per event, and the intensive observable $p_{T}$, the mean transverse momentum per event. We first describe all three effects briefly, and then describe the simulation which we have used in order to investigate them. The first (and largest) effect is a direct contribution to the fluctuations of $N$, and indeed to any extensive observable. The multiplicity fluctuations in a classical ideal gas are characterized by $\left\langle(\Delta N)^{2}\right\rangle /\langle N\rangle=1$ and for an ideal pion gas this ratio is $1+(1 / 2){\overline{\left\langle n_{p}\right\rangle}}^{\text {inc }}$ due to Bose effects and is therefore a few percent larger than 1 . This ratio is significantly larger for a resonance gas. Each resonance decays into several pions (for example, $\rho \rightarrow 2 \pi, \omega, \eta \rightarrow 3 \pi$, etc), and this means that when the resonances decay after freeze-out, they significantly modify the statistics of pion number fluctuations. If the resonances themselves are produced randomly】, with a Poisson multiplicity distribution, their decay products are not. For example, if there were no direct pions and only one species of resonance which always decayed into $d$ charged pions, the pions produced in this ensemble would have $\left\langle(\Delta N)^{2}\right\rangle /\langle N\rangle=d$.

Resonances also affect the fluctuations of intensive observables, like the energy per pion or mean transverse momentum $p_{T}$. The second effect we analyze arises because pions produced in resonance decays have a single-particle momentum spectrum which is similar but not identical to the thermal spectrum for the direct pions. The products of resonance decay populate the low $p_{T}$ region of the spectrum somewhat more. In order to estimate $v_{\text {ebe }}\left(p_{T}\right)$, we must therefore include the change in $v_{\text {inc }}\left(p_{T}\right)$ introduced by the pions produced by the decay of resonances after freeze-out.

The third effect of resonance decays is that they contribute additional kinematic correlations between their decay products, which then have no chance to rethermalize. New terms arise in the correlator (7) which describes the fluctuations microscopically. For example, two-body decay (such as $\rho \rightarrow \pi^{+} \pi^{-}$at rest) generates a term in the correlation function:

$$
\left\langle\Delta n_{p}^{+} \Delta n_{k}^{-}\right\rangle=\delta_{p,-k} C(p),
$$

\footnotetext{
${ }^{6}$ Note that this number is close to the critical temperature obtained in lattice simulations with zero baryon density 24.

${ }^{7}$ All resonances are heavy enough that Bose enhancement for them can be neglected.

${ }^{8}$ This effect is qualitatively similar to the effect of a nonzero pion chemical potential. A clear distinction between these effects in data analysis is still lacking.
} 
where $C(p)$ is proportional to the square of the pion fraction originating from $\rho_{0}$ decays. The result of all such terms will be a change in $v_{\text {ebe }}\left(p_{T}\right)$ which can be parameterized as a new contribution $F_{\text {res }}$ to the ratio $F$ of (25). (That is, we now have $F=F_{B} F_{\text {res }}$.) Instead of attempting to study all contributions like (31) one by one, we address this effect and the first two by doing a simulation. We will see that the second and third effects we have described are both small.

We have simulated a gas of pions, nucleons and resonances in thermal equilibrium at freeze-out, including the $\pi, K, \eta, \rho, \omega, \eta^{\prime}, N, \Delta, \Lambda, \Sigma$ and $\Xi$, and then simulated the subsequent decay of the resonances. That is, we have generated an ensemble of pions in three steps: (i) Thermal ratios of hadron multiplicities were calculated assuming equilibrium ratios at chemical freeze-out. Following [23], the values $T_{\mathrm{ch}}=170 \mathrm{MeV}$ and $\mu_{\text {baryon }}=200$ $\mathrm{MeV}$ were used. (ii) Then, a program generates hadrons with multiplicities determined at chemical freezeout, but with thermal momenta as appropriate at the thermal freeze-out temperature, which we take to be $T_{\mathrm{f}}=120 \mathrm{MeV}$, with $\mu_{\pi}=60 \mathrm{MeV}$. The last step (iii) is to decay all the resonances, using the appropriate subroutine from RQMD.] Under these conditions, more than half of the observed pions come from resonance decays.

We evaluate the variance of the fluctuations of the multiplicity of the pions obtained from the resonance gas as follows. For each species in the resonance gas, we label the different decay modes by an index $i$, and refer to the branching ratios for the species $r$ as $b_{r}^{i}$. For each decay we define $d_{r}^{i}$, the number of charged pions produced. From the simulation, we obtain the multiplicity of each resonance, $N_{r}$. The total multiplicity of pions is $N_{\pi}=\sum_{r, i} d_{r}^{i} b_{r}^{i} N_{r}$ and the multiplicity fluctuations are described by

$$
\frac{\left\langle\left(\Delta N_{\pi}\right)^{2}\right\rangle}{\left\langle N_{\pi}\right\rangle}=\frac{\sum_{r, i}\left(d_{r}^{i}\right)^{2} b_{r}^{i} N_{r}}{\sum_{r} d_{r}^{i} b_{r}^{i} N_{r}} \approx 1.4 .
$$

Bose enhancement increases this to $\left\langle\left(\Delta N_{\pi}\right)^{2}\right\rangle /\left\langle N_{\pi}\right\rangle \approx 1.5$. W We see that the resonances increase the multiplicity fluctuations by a large factor, relative to the fluctuations of the direct pions alone. We compare this result to what is seen in NA49 data below.

We now turn to the resonance-induced contribution to the fluctuations of the intensive observable $p_{T}$, which is much smaller. We begin with the effect on $v_{\text {inc }}$. Table 1 describes the single-particle inclusive distribution obtained from the simulation, assuming uncorrelated particles in an equilibrium resonance gas at freeze-out. We see that the resonances change $v_{\text {inc }}\left(p_{T}\right) /\left\langle p_{T}\right\rangle$ only by a few percent. The contributions of correlations induced by resonance decay and of Bose enhancement to $F$ are not included. The effects of flow are not included. We now discuss each in turn, and find that all yield small contributions to $\langle N\rangle^{1 / 2} v_{\text {ebe }}\left(p_{T}\right) /\left\langle p_{T}\right\rangle$ relative to $v_{\text {inc }}\left(p_{T}\right) /\left\langle p_{T}\right\rangle$ which we have evaluated in the table.

We have estimated $F_{\text {res }}$ by slicing up the pions from Table 1 into varying numbers (up to 2500) of events, and evaluating $F$. Since Bose enhancement is not included in the simulation,

\footnotetext{
${ }^{9}$ We treat particles which decay by weak interactions as stable, which raises an additional issue. Experimentally, some weak decays happen so quickly that they feed up into the observed pion spectra. We treat these particles as stable here; we hope that experimentalists make the appropriate corrections to the data.

${ }^{10}$ Event-by-event fluctuation in the resonance multiplicities $N_{r}$, as may be computed, for example, in dynamical models in which the resonances themselves are produced by decays of "clusters", may result in a small further increase in this ratio.
} 


\begin{tabular}{|l||c|c|c|c|}
\hline & $\begin{array}{c}\text { no. of pions, } \\
10^{3}\end{array}$ & $\begin{array}{c}\left\langle p_{T}\right\rangle, \\
\mathrm{MeV}\end{array}$ & $\begin{array}{c}v_{\text {inc }}\left(p_{T}\right), \\
\mathrm{MeV}\end{array}$ & $v_{\text {inc }}\left(p_{T}\right) /\left\langle p_{T}\right\rangle$ \\
\hline "direct pions" only & 541 & 283 & 189 & 0.67 \\
\hline pions from resonances only & 713 & 271 & 177 & 0.65 \\
\hline all pions & 1254 & 276 & 183 & 0.66 \\
\hline
\end{tabular}

Table 1: Results of a numerical simulation of a resonance gas. The results include the effects of the the correlations induced by resonance decays on the inclusive $p_{T}$ spectrum. The simulation itself does not include Bose enhancement effects, and so can be thought of as the simulation of a single event with $1.254 \cdot 10^{6}$ pions with a mean $p_{T}$ of $276 \mathrm{MeV}$, or can be sliced up into smaller events.

the $F$ so obtained is just $F_{\text {res }}$. We find no statistically significant contribution to $F$, and conclude that $\left|F_{\text {res }}-1\right|<0.01$.

We now use the results of Section 3.1 to incorporate Bose enhancement effects, after noting the connection between Bose enhancement and resonance decay pions. There can be quantum interference between direct pions and resonance decay pions, or among resonance decay pions. It is well known that all resonances can be approximately separated into two groups: those which are short-lived and those which are long-lived. The former (e.g., $\rho$ and $\Delta$ ) have lifetimes much shorter than the duration of pion radiation from the fireball (i.e., the time over which freeze-out occurs). Therefore, their decay products interfere with other pions. The decay products of long-lived particles (e.g., $\omega$ and $\eta$ ) can only interfere with other pions if one selects pions with a very small energy difference $\left|\omega_{1}-\omega_{2}\right|<<\Gamma$, where $\Gamma$ is the width of the resonance. This is essentially impossible, and pions produced in the decay of long-lived particles therefore do not contribute to the Bose enhancement factor. This means that $F_{B}-1$ should be multiplied by a factor $\left(f_{\text {direct }}+f_{\rho}+f_{\Delta}+\ldots\right)^{2}$ where the $f$ 's are the fractions of all $\pi$ mesons coming from short-lived sources. This same fraction enters the HBT correlation function, and is about 0.5. 25] So, we take $F_{B}-1=0.073$ for $\mu_{\pi}=60 \mathrm{MeV}$ from Fig.(四) and reduce it by a factor of 0.5 yielding

$$
F_{B}=1.037
$$

and therefore conclude that the effect of Bose enhancement is a small increase in $v_{\text {ebe }}\left(p_{T}\right)$ by a factor of $\sqrt{F_{B}}=1.018$.

\subsection{The Effects of Radial Flow}

To this point, we have calculated the fluctuations in $p_{T}$ as if the matter in a heavy ion collision were at rest at freeze-out. This is not the case: by that stage the hadronic matter is undergoing a collective hydrodynamic expansion in the transverse direction, and this must be taken into account in order to compare our results with the data. A very important point here is that the fluctuations in pion multiplicity are not affected by flow, and our prediction for them is therefore unmodified. Fluctuations in multiplicity ratios (e.g. $K / \pi$ ) would also be unaffected. However the event-by-event fluctuations of mean $p_{T}$ are certainly affected by flow. The fluctuations we have calculated pertain to the rest frame of the matter at 
freeze-out, and we must now boost them. A detailed account of the resulting effects would require a complicated analysis. Here we shall use the simple approximation [26] that the effects of flow on the pion momenta can be treated as a Doppler blue shift of the spectrum: $n\left(p_{T}\right) \rightarrow n\left(p_{T} \sqrt{1-\beta} / \sqrt{1+\beta}\right)$. This blue shift increases $\left\langle p_{T}\right\rangle$, and increases $v_{\text {inc }}\left(p_{T}\right)$, but leaves the ratio $v_{\text {inc }}\left(p_{T}\right) /\left\langle p_{T}\right\rangle$ (and therefore the ratio $v_{\text {ebe }}\left(p_{T}\right) /\left\langle p_{T}\right\rangle$ ) unaffected. This ratio (the fourth column in Table 1) is therefore a good quantity to compare to experimental data, since our goal here is to extract information about thermodynamics and not about flow.

However, event-by-event fluctuations in the flow velocity $\beta$ must still be taken into account. This issue was discussed qualitatively already in [11], where it was argued that this effect must be relatively weak. Here we provide the first rough estimate of its magnitude. The magnitude of the flow velocity is proportional to the integral of the pressure gradient over the expansion time. Thus the fluctuations of the flow velocity are determined by the pressure fluctuations. The size of the pressure fluctuations is related to the adiabatic compressibility by the standard thermodynamic relation [18]

$$
\left\langle(\Delta P)^{2}\right\rangle=-T\left(\frac{\partial P}{\partial V}\right)_{S} .
$$

For the resonance gas equation of state this gives

$$
\frac{\left\langle(\Delta P)^{2}\right\rangle}{P^{2}}=\frac{\kappa}{\kappa-1} \frac{T}{P V}=\frac{\kappa^{2}}{\kappa-1} \frac{1}{S} \text {. }
$$

The entropy per pion in the ideal gas is around 2.4, and is larger for the resonance gas. We shall take $S \approx 3 N_{\pi}$ for our estimate.

Using the "blue shift" approximation we can write

$$
p_{T} \approx p_{T}^{\text {rest }} \sqrt{\frac{1+\beta}{1-\beta}},
$$

where $p_{T}^{\text {rest }}$ is the corresponding momentum in the rest frame of the matter. The fluctuations of the observed momentum are then related to the fluctuations in the rest frame (calculated above) and the flow velocity fluctuations through

$$
\frac{\left\langle\left(\Delta p_{T}\right)^{2}\right\rangle}{p_{T}^{2}}=\frac{\left\langle\left(\Delta p_{T}^{\text {rest }}\right)^{2}\right\rangle}{\left(p_{T}^{\text {rest }}\right)^{2}}+\left\langle(\Delta \beta)^{2}\right\rangle,
$$

where we have neglected corrections which are suppressed by $O(\beta)$ relative to $\left\langle(\Delta \beta)^{2}\right\rangle$. The fluctuations in the flow velocity are given by

$$
\left\langle(\Delta \beta)^{2}\right\rangle=\beta^{2} \frac{\left\langle(\Delta P)^{2}\right\rangle}{P^{2}}\left(\frac{\tau_{\text {micro }}}{\tau_{\text {flow }}}\right) .
$$

The last factor on the right-hand side appears because the final velocity is proportional to the time integral of the pressure gradient over the entire evolution prior to freeze-out, and this integral is a sum over uncorrelated fluctuations in time. In a resonance gas one can discuss the typical duration of a collision (the lifetime of a typical resonance), and the time between collisions (the inverse of the scattering rate). Both are close to the "microscopic" 
time scale $\tau_{\text {micro }} \sim 1 \mathrm{fm} / c$. The expansion duration relevant for radial flow is actually much longer, $\tau_{\text {flow }} \approx 10-20 \mathrm{fm} / c$ for central $\mathrm{Pb}-\mathrm{Pb}$ collisions. This means that for each microscopic volume element one first does the time integral and obtains a "random walk" factor $\left(\tau_{\text {micro }} / \tau_{\text {flow }}\right)^{1 / 2} \sim 1 / 4$ in $\Delta \beta$. Then, the sum over uncorrelated volume elements leads to a $1 / \sqrt{V}$ or $1 / \sqrt{N}$, which we have already seen in the expression (35) for $\langle\Delta P\rangle$.

The flow velocity can be estimated for our purposes from the ratio of $\left\langle p_{T}^{\text {rest }}\right\rangle \approx 276 \mathrm{MeV}$ given in Table 1 and the experimental $\left\langle p_{T}\right\rangle \approx 376 \mathrm{MeV}$ observed by NA49. Thus, $\beta \approx 0.3$. Finally, putting all the estimates into eq. (38) we find

$$
N\left\langle(\Delta \beta)^{2}\right\rangle \approx(0.1)^{2}
$$

Note that although our estimate is uncertain at various points, the result is very small. Even if we have underestimated the size of $\left\langle(\Delta \beta)^{2}\right\rangle$ by a factor of 4 , the contribution to $v_{\text {inc }}\left(p_{T}\right) /\left\langle p_{T}\right\rangle$ would only be 0.02 . It is quite clear that the great bulk of $v_{\text {inc }} /\left\langle p_{T}\right\rangle$ is thermodynamic, with the contributions of the fluctuations in the flow velocity being negligible in comparison.

The largest uncertainty in our estimate for $v_{\text {inc }}\left(p_{T}\right) /\left\langle p_{T}\right\rangle$ is not due to the fluctuations in the flow velocity, which can clearly be neglected, but is due to the velocity itself. The blue shift approximation which we have used applies quantitatively only to pions with momenta greater than their mass 26]. Because of the nonzero pion mass, boosting the pions does not actually scale the momentum spectrum by a momentum independent factor. Furthermore, in a real heavy ion collision there will be a position dependent profile of velocities, rather than a single velocity $\beta$. A more complete calculation of $v_{\text {inc }}\left(p_{T}\right) /\left\langle p_{T}\right\rangle$ would require a better treatment of these effects in a hydrodynamic model; we leave this for the future.

We obtain our final estimate of the magnitude of the event-by-event fluctuations of the intensive quantity $p_{T}$ far from the critical point as follows. Using the estimate of

$v_{\text {inc }}\left(p_{T}\right)^{\text {rest }} /\left\langle p_{T}\right\rangle^{\text {rest }}$ from Table 1 and Eqs. (37,39), we estimate that fluctuations in the flow velocity increase $v_{\text {inc }}\left(p_{T}\right) /\left\langle p_{T}\right\rangle$ from 0.66 to 0.67 . Multiplication by $\sqrt{F_{B}}$ then yields

$$
\frac{\langle N\rangle^{1 / 2} v_{\mathrm{ebe}}\left(p_{T}\right)}{\left\langle p_{T}\right\rangle} \approx 0.68,
$$

subject to the uncertainties introduced by the blue shift approximation.

\subsection{Comparison with NA49 Data and Outlook}

In this section we compare our results with the NA49 data from central Pb-Pb collisions [7] summarized in Table 2. As a first qualitative check of the predictions of our resonance gas model, we can look at the multiplicity fluctuations. It is clear that with no cut on centrality, one would see a very wide non-Gaussian distribution of multiplicity determined by the geometric probability of different impact parameters $b$. Gaussian thermodynamic fluctuations can only be seen if a tight enough cut in centrality is applied. The event-byevent $N$-distribution found by NA49 when they use only the $5 \%$ most central of all events, with centrality measured using a zero degree calorimeter, is Gaussian to within about $5 \%$. This cut corresponds to keeping collisions with impact parameters $b<3.5 \mathrm{fm}$. [7] The nonGaussianity could be further reduced by tightening the centrality cut further. We now ask 


\begin{tabular}{|l||l|}
\hline number of events & 98426 \\
\hline total number of charged particles & 26587685 \\
\hline$\langle N\rangle$ & $270.13 \pm 0.07$ \\
\hline$v_{\text {ebe }}(N)$ & $23.29 \pm 0.05$ \\
\hline$\left\langle p_{T}\right\rangle$ & $376.75 \pm 0.06 \mathrm{MeV}$ \\
\hline$v_{\text {inc }}\left(p_{T}\right)$ & $282.16 \pm 0.04 \mathrm{MeV}$ \\
\hline$v_{\text {ebe }}\left(p_{T}\right)$ & $17.27 \pm 0.03 \mathrm{MeV}$ \\
\hline
\end{tabular}

Table 2: Preliminary NA49 data[7]. The charged particles are taken from the kinematic region $0.005<p_{T}<2 \mathrm{GeV}$ and $4<y<5.5$ (assuming $\pi$ mass). The events used are the $5 \%$ most central of all events, with centrality measured using a zero degree calorimeter. The products from weak decays such as $\Lambda^{\text {'s }}$ and $K^{0}$ 's, were only partially rejected with approximately $60 \%$ rejection efficiency. The errors are statistical only.

how well our resonance gas describes the width of the (almost) Gaussian distribution. From the data, we have

$$
\frac{v_{\mathrm{ebe}}^{2}(N)}{\langle N\rangle}=2.008 \pm 0.009,
$$

which we should compare to our resonance gas prediction of 1.5. 1 We therefore conclude that about $75 \%$ of the observed fluctuation is thermodynamic in origin. The contamination introduced into the data by fluctuations in centrality could be reduced by analyzing data samples with more or less restrictive cuts but the same $\langle N\rangle$, and extrapolating to a limit in which the cut is extremely restrictive. This could be done using cuts centered at any centrality. In addition to fluctuations in centrality, there is another experimental (as opposed to thermodynamic) factor which could affect the agreement between resonance gas predictions and the observed fluctuations. The increase in the fluctuations due to resonances can only be detected provided the detector acceptance is large enough to ensure the detection of all (or most) of the decay products. NA49 seem to have coverage wide enough to satisfy this criterion and a quantitative estimate of losses on its boundaries can easily be made. Our resonance gas model predicts that as the centrality cut is tightened, the ratio $v_{\text {ebe }}^{2}(N) /\langle N\rangle$ should decrease toward a limit near 1.5.

Although further work is certainly required, it is already apparent that the bulk of the multiplicity fluctuations observed in the data are thermodynamic in origin. Because the multiplicity fluctuations are sensitive to impact parameter fluctuations, it may prove difficult to explain their magnitude with greater precision even in future. However, the fact that they are largely thermodynamic in origin suggests that the effects present near the

\footnotetext{
${ }^{11}$ In the NA49 data of Table 2, all charged particles are counted whereas we have done our calculations assuming that only the charged pions are observed. In our resonance gas model and in the data[7], about $80 \%$ of the charged particles in the final state are pions. If we redo the calculation (32), but this time define $d_{r}^{i}$ as the number of charged particles (pions, kaons, protons) produced in the $i$ 'th decay of the $r$ 'th resonance, we find that that $v_{\text {ebe }}^{2}(N) /\langle N\rangle$ increases, but only by a few percent. NA49 has demonstrated that it can study particle identification event-by-event and it may therefore be possible to analyze data on charged pion multiplicity in future.
} 
critical point, which we study in Sections 5 and 6, could result in a significant nonmonotonic enhancement of the multiplicity fluctuations. This would be of interest whether or not the noncritical fluctuations on top of which the nonmonotonic variation occurs are understood with precision.

Now, we proceed to $p_{T}$ fluctuations. As we explain in the Appendix, in order to be sure that $F=1$ when there are no correlations between pions, care must be taken in constructing an estimator for $v_{\text {ebe }}\left(p_{T}\right)$ using a finite sample of events, each of which has finite multiplicity. The appropriate prescription (145) is to weight events in the event-by-event average by their multiplicity. This has not been done in Table 2. However, we show in (147) that we can use $\langle N\rangle$ and $v_{\text {ebe }}(N)$ to change $v_{\text {ebe }}\left(p_{T}\right)$ as required, and the result is

$$
v_{\text {ebe }}\left(p_{T}\right)=(17.27 \pm 0.03 \mathrm{MeV})\left(1-\frac{1}{2} \frac{v_{\text {ebe }}^{2}(N)}{\langle N\rangle^{2}}\right)=(17.21 \pm 0.03 \mathrm{MeV}) .
$$

We use this henceforth. We must now compare

$$
\frac{\langle N\rangle^{1 / 2} v_{\text {ebe }}\left(p_{T}\right)}{\left\langle p_{T}\right\rangle}=0.751 \pm 0.001
$$

to our prediction (40) of 0.68 .

We see that the major part of the observed fluctuation in $p_{T}$ is accounted for by the thermodynamic fluctuations we have considered. A large part of the discrepancy is in our prediction for the variance of the inclusive single-particle distribution $v_{\text {inc }}\left(p_{T}\right)$. Our $v_{\text {inc }}\left(p_{T}\right) /\left\langle p_{T}\right\rangle=0.67$ is about $10 \%$ lower than that in the data. ${ }^{12}$ First, this suggests that there may be a small nonthermodynamic contribution to the $p_{T}$-fluctuations, for example from fluctuations in the impact parameter. The other source of this discrepancy is the blue shift approximation. We have applied a blue shift factor such that $\left\langle p_{T}\right\rangle$ increases from $281 \mathrm{MeV}$ in Table 1 to $377 \mathrm{MeV}$ as in the data, and in so doing have obtained a value for $v_{\text {inc }}\left(p_{T}\right)$ which is low by $10 \%$. This may be a reasonable estimate for the error which we have introduced by using the blue shift approximation rather than a more sophisticated treatment of the effects of flow on the spectrum, which we leave to future work. Such a treatment is necessary before we can estimate how much of the $10 \%$ discrepancy is introduced by the blue shift approximation. Future work on the experimental side (varying the centrality cut) could lead to an estimate of how much of the discrepancy is due to impact parameter fluctuations.

We have gone as far as we will go in this paper in our quest to understand the thermodynamic origins of the width of the inclusive single particle distribution. Another very

\footnotetext{
${ }^{12}$ As we have already noted, all charged particles are included in the data whereas we have calculated the fluctuations for the charged pions alone. We have checked that including the protons and charged kaons from the resonance gas increases our prediction for $v_{\text {inc }}\left(p_{T}\right) /\left\langle p_{T}\right\rangle$ in the rest frame by only a few percent. This small increase in the ratio is likely further reduced once the flow-induced increase in $\left\langle p_{T}\right\rangle$ for the kaons and protons is taken into account. Although it would be good to remove this uncertainty completely by analyzing a data sample of pions alone, it is already clear that this is not the explanation for the present $10 \%$ discrepancy.

${ }^{13}$ We expect that the fluctuations of an intensive quantity like $p_{T}$ are less sensitive to impact parameter fluctuations than are those of the multiplicity, and this seems to be borne out by the data.
} 
important feature in the data is the value of the ratio of the scaled event-by-event variation to the variance of the inclusive distribution:

$$
\sqrt{F}=\frac{\langle N\rangle^{1 / 2} v_{\text {ebe }}\left(p_{T}\right)}{v_{\text {inc }}\left(p_{T}\right)}=1.002 \pm 0.002 .
$$

The difference between the scaled event-by-event variance and the variance of the inclusive distribution is less than a percent. This is a remarkable fact, since the contribution of the Bose enhancement (see Section 3.1) to this difference is almost an order of magnitude bigger $\left(\sqrt{F_{B}}-1\right.$ is a few percent). Therefore, there must be some mechanism at work which compensates for the Bose enhancement. One possible mechanism is the effect of the twotrack resolution, diminishing the observed number of pions with very similar momenta[0]. This reduces the ratio $F$, and NA49 estimates that it is of comparable magnitude to the Bose enhancement effect but with the opposite sign. We do not attempt to include either this effect or the effect of final state Coulomb interactions between charged pions in our analysis, leaving that to the experimentalists. However, we point out that in the next section we find another possible origin of this effect. We shall see that anticorrelations due to energy conservation and thermal contact between the observed pions and the rest of the system reduce $F$, as long as the system does not freeze out near the critical point.

In summary, we have shown in this section that the assumption that the system is a thermal resonance gas at freeze-out is in reasonable agreement with the magnitude of the observed event-by-event fluctuations in the pion multiplicity and mean $p_{T}$. We will see in Section 4 that the effects of energy conservation bring our prediction for $\sqrt{F}$ into even better agreement with the data. Of course, a number of issues we have touched upon need further study: it cannot be otherwise for the first quantitative study of a new set of phenomena. The situation is, however, very encouraging. First, RHIC detectors are very well-suited to measurements of the fluctuations we have analyzed. Second, although some of the interesting effects are small ( $F_{B}$, for example) with millions of recorded events all Gaussian widths can be measured to much better statistical accuracy than even the smallest of the systematic effects we have discussed, and will discuss in later sections. Third, the interesting systematic effects can be studied by varying the cuts made on the data. For example, considering only lowmomentum pions one should find the effect of both the resonances and the Bose enhancement (see Fig.(11) ) to be several times higher. Also, $\mu_{\pi}$ and therefore the Bose enhancement factor $F_{B}$ may be somewhat larger in central events. Detailed study (varying centrality; varying cuts in $p_{T}$ ) may allow experimentalists to separate the effects of Bose enhancement from other effects we have described, and will describe later in this paper. Fourth, one can significantly widen the types of fluctuations which are analyzed. For example, one can study new correlators like the event-by-event cross correlation between multiplicity and mean $p_{T}$, $\left\langle\Delta p_{T} \Delta N\right\rangle$. We saw in Section 2 that such cross correlation results only from nontrivial effects. Finally, it is important to note that we do not expect any of the effects we have analyzed in this Section to change significantly near the critical point.

\footnotetext{
${ }^{14}$ As noted above, because $v_{\text {inc }}\left(p_{T}\right)$ is scaled by the blue shift introduced by the expansion velocity, so is $\Phi_{p_{T}}$. This makes $\Phi_{p_{T}}$ harder to predict than $F$. However, for convenience, we note that if one uses the experimental value of $v_{\text {inc }}\left(p_{T}\right)$, a value $\sqrt{F}=1.01$ corresponds to $\Phi_{p_{T}}=2.82 \mathrm{MeV}$, and the $\sqrt{F}$ in the data (44) corresponds to $\Phi_{p_{T}}=0.6 \pm 0.6 \mathrm{MeV}$.
} 
Our analysis demonstrates that the observed fluctuations are broadly consistent with thermodynamic expectations, and therefore raises the possibility of large effects when control parameters are changed in such a way that thermodynamic properties are changed significantly, as at a critical point. The smallness of the statistical errors in the data also highlights the possibility that many of the interesting systematic effects we analyze in this paper will be accessible to detailed study as control parameters are varied.

\section{Using an Ideal Gas of Pions as a Thermometer}

To this point, we have assumed that the system does not freeze out close to the critical point, and can be approximated at freeze-out as a non-interacting ideal resonance gas. In this Section, we take a first step towards understanding how the physics characteristic of the vicinity of the critical point affects the event-by-event fluctuations. Along the way, we quantify the effects of energy conservation on the $p_{T}$-fluctuations. This leads to a small

reduction in $\sqrt{F}$ far from the critical point, which may be required by the data (44). In this Section, we consider only the "direct pions", and as before we treat them as an ideal Bose gas at freeze-out. We further imagine that the pions are in thermal contact with the "rest of the system", which is not directly observed and which need not be ideal. The rest of the system includes the neutral pions, the resonances, the pions not in the experimental acceptance and, most important, the order parameter or sigma field. If freeze-out occurs in the vicinity of the critical point, the thermodynamic properties of the sigma field (and therefore of "the rest of the system") are singular. In the analysis of this section, we imagine that the observed pions are an ideal gas even for freeze-out in the vicinity of the critical point, while the equation of state and susceptibilities of the rest of the system become singular there. Some of the universal critical indices characterizing this singularity are discussed in [1]. The question we ask here is how the fluctuations of the pions are affected by being in thermal contact with the rest of the system, particularly when the susceptibilities characterizing the sigma field diverge.

A reader who is used to thinking about the $O(4)$ second order transition may be concerned that we are treating the pions and the sigma field so differently. The point is that near the critical endpoint which we wish to analyze (and which may occur in nature) the pions and sigma are different. The pions remain massive, while the sigma mass vanishes and the long wavelength modes of the sigma field undergo critical fluctuations and are almost classical. The divergence of the specific heat of the system as a whole is primarily due to the fluctuations of the sigma field. The analysis of this section is therefore a reasonable first step. What it leaves out, of course, is the fact that the pions, although not massless, do interact strongly with the sigma field and are therefore not an ideal gas. We are neglecting the direct effects of the pion-sigma coupling. Once these are included, it is not possible to make a clean separation between ideal pions and singular rest of the system. We analyze the consequences of the pion-sigma coupling in Section 5. 


\subsection{Thermometers, Temperature Fluctuations and Heat Capacity}

Let us step back and recall the text-book formalism describing the measurement of temperature. A thermometer should be a simple system which has been already calibrated, in the sense that we can relate its total energy $E$ to its temperature $T$ via a function $T(E)$ which we already know. Instead of $E$, we could also use any other mechanical observable, like for example the volume of the liquid in a liquid thermometer. An ideal pion gas makes a very good thermometer because it is a simple system with a known equation of state. Having the equation of state, we read off $T$ by measuring a mechanical observable, such as $E$.

If the mechanical observable fluctuates, so will the measured temperature. In particular, if we measure the total energy, which fluctuates as $\left\langle(\Delta E)^{2}\right\rangle=T^{2} C_{V}$ in the canonical ensemble the temperature $T(E)$ will also fluctuate, with $\left\langle(\Delta T)^{2}\right\rangle$ given by (11). Note that given that we measure a mechanical observable $E$ rather than $T$, in order to find the result (1) we must know $T(E)$. This is possible for a thermometer like an ideal pion gas, but may not be possible for the system one wishes to study using the thermometer. One of the questions we address in this section is when we measure the energy of the thermometer only (instead of measuring the energy of the whole system) which $C_{V}$ is relevant: that of the thermometer, that of the rest of the system, or a combination.

Suppose now we use our thermometer $B$ to measure the temperature of another system $A$. The measurement consists of bringing the two systems in thermal contact. If the resulting system $A+B$ is closed, thermal equilibrium will result. By ergodicity, the thermodynamic ensemble will consist of all the states with the same energy, $E_{A+B}$, taken with equal probability weight. Although the total energy does not fluctuate, the energies of the subsystems $E_{A}$ and $E_{B}$ do, subject to a constraint $E_{A}+E_{B}=E_{A+B}$. The probability that the subsystem $B$ has energy $E_{B}$ is proportional to the number of states, $\Gamma$, of the system $B$ with energy $E_{B}$ times the number of states of $A$ with energy $E_{A}=E_{A+B}-E_{B}$ :

$$
\Gamma_{A+B}\left(E_{A+B}\right)=\sum_{E_{B}} \Gamma_{A}\left(E_{A+B}-E_{B}\right) \Gamma_{B}\left(E_{B}\right) .
$$

Both $\Gamma_{A, B}$ are exponentially growing functions of their arguments (and also the size of the system) and their product on the right-hand side of (45) has a sharp maximum at some value of $E_{B}$. Introducing the entropy $S$ as $S(E)=\ln \Gamma(E)$, we can write for the value of $E_{B}$ at the maximum:

$$
0=\frac{d}{d E_{B}}\left[S_{A}\left(E_{A+B}-E_{B}\right)+S_{B}\left(E_{B}\right)\right]=-\frac{d S_{A}}{d E_{A}}+\frac{d S_{B}}{d E_{B}}=-\frac{1}{T_{A}}+\frac{1}{T_{B}},
$$

since the temperature is, by definition, $1 / T=d S / d E$. We recover the text-book result that the temperatures of two systems in equilibrium are equal. Measuring $E_{B}$, and using the known function $T_{B}\left(E_{B}\right)$, we find this (common) temperature.

Of course, it is not necessary that the system $A+B$ is rigorously closed. In practice it is sufficient that the rate of the thermal equilibration between $A$ and $B$ is faster than the rate of thermal equilibration of $A+B$ with the environment.

So far we have only discussed the mean value of $E_{B}$ and, consequently, the mean temperature. The size of the fluctuations of $E_{B}$ is given by the width of the maximum in 
$\Gamma_{A}\left(E_{A+B}-E_{B}\right) \Gamma_{B}\left(E_{B}\right)$. We need the second derivative:

$$
\frac{d^{2}}{d E_{B}^{2}}\left[S_{A}\left(E_{A+B}-E_{B}\right)+S_{B}\left(E_{B}\right)\right]=\frac{d^{2} S_{A}}{d E_{A}^{2}}+\frac{d^{2} S_{B}}{d E_{B}^{2}}=-\frac{1}{T^{2}}\left(\frac{1}{C_{A}}+\frac{1}{C_{B}}\right),
$$

where $C_{A, B}$ are the heat capacities of the systems $A$ and $B$. Thus we find for $\Delta E=\Delta E_{B}=$ $-\Delta E_{A}$ :

$$
\left\langle(\Delta E)^{2}\right\rangle=T^{2}\left(\frac{1}{C_{A}}+\frac{1}{C_{B}}\right)^{-1} .
$$

The importance of the result (48) is that the thermometer $B$ allows us not only to measure the temperature of the system $A$, but also the heat capacity of the system $A !^{\mathrm{P}}$ In order to make such a measurement, we must watch the fluctuations of $E_{B}$ in addition to $\left\langle E_{B}\right\rangle$.

Another consequence of (48) is that when $C_{A} \gg C_{B}$ we recover the result for the canonical ensemble (11). What is important is that the heat capacity $C_{V}$ appearing in (11) in this case is that of the thermometer itself, $C_{B}$, and not that of the measured system, $C_{A}$.

Now, suppose that the system $A$ has a thermodynamic singularity at some temperature, as a result of which $C_{A} \rightarrow \infty$. This is precisely the situation which arises near the critical point in the idealization of this section: the ideal pion thermometer $B$ is in thermal contact with a system $A$ with divergent susceptibilities. Equation (48) tells us that the fluctuations of the energy, which are equal in $A$ and $B$ due to the conservation of energy in $A+B$, will increase as we approach the critical point where $C_{A}$ diverges. What happens to the temperature fluctuations? Remember, that we do not measure the temperature directly, but use the equation of state $T(E)$ to read it off from the value of $E$. If we used the equation of state of the system $A, T_{A}\left(E_{A}\right)$, the fluctuations of $T_{A}$ would decrease and vanish at the critical point as discussed in [1], because $C_{A}=d E_{A} / d T_{A}=\infty$. However, the equation of state of the system $A$ is not known to us. Indeed, we are trying to learn about it doing our measurements. If we instead use the equation of state of the thermometer $T_{B}\left(E_{B}\right)$ which is nonsingular, the fluctuations of $T_{B}$ will increase because the fluctuations of $E$ do, and will approach the value determined by (1) with $C_{V}=C_{B}$.

Note that the temperatures of the systems $A$ and $B$ determined through their respective equations of state are different on the event-by-event basis. This is not in contradiction with thermodynamics which only requires the mean values to agree as in (46).

Returning to our idealized system at freeze-out we want to use pions we observe as a thermometer, $B$. The rest of the system, which includes all the other particles, including pions not ending up in our detectors, we consider as system $A$. The singularity of the heat capacity occurs in $C_{A}$, while $C_{B}$ is the heat capacity of the ideal gas and is regular. Nevertheless this singularity affects the fluctuations of the pions through (48). The effect of the singularity in $C_{A}$ is an increase in the fluctuation $\left\langle(\Delta E)^{2}\right\rangle$. If one were able to use $T_{A}(E)$ to define $T$, one would find that fluctuations in $T_{A}$ would decrease at the critical point. Using $T_{B}(E)$, or any practical definition of a temperature, leads to fluctuations in $T$ which, like those in $E$, increase. Since what we measure is always a mechanical thermodynamic observable, like the total energy $E$, or the energy per particle, or the transverse momentum

\footnotetext{
${ }^{15}$ One example of such a measurement in a simple lattice system can be found in 27.
} 
per particle, etc., it is not in fact necessary to do a translation to the temperature variable to detect a singularity. It is easier to look directly at the fluctuations of observable quantities. To this we now turn.

\subsection{The Microscopic Correlator}

As discussed in Section 2.1, the mean square variations of thermodynamic observables in the pion gas are determined by the microscopic correlator: $\left\langle\Delta n_{p} \Delta n_{k}\right\rangle$. Once we find this correlator we can then use it to calculate any fluctuations of interest.

For the case of the canonical ensemble this correlator is given by (7) which leads to:

$$
\left\langle(\Delta E)^{2}\right\rangle=\sum_{p} \epsilon_{p}^{2} v_{p}^{2}=T^{2} C_{B}
$$

as in (10) and (14). This corresponds to the case $C_{A}=\infty$, where $B$ is a (grand) canonical ensemble. In the case when $C_{A}$ is finite (48) tells us that the correlator $\left\langle\Delta n_{p} \Delta n_{k}\right\rangle$ should change. A simple derivation of this correlator given below yields the result

$$
\left\langle\Delta n_{p} \Delta n_{k}\right\rangle=v_{p}^{2} \delta_{p k}-\frac{v_{p}^{2} \epsilon_{p} v_{k}^{2} \epsilon_{k}}{T^{2} C_{A}+\sum_{p} v_{p}^{2} \epsilon_{p}^{2}} .
$$

This result is easy to understand intuitively and it passes many nontrivial checks. When $C_{A} \gg C_{B}=\sum_{p} \epsilon_{p}^{2} v_{p}^{2} / T^{2}$ the second term in (50) is negligible and we recover (7). On the other hand, when $C_{A}=0$, the system $B$ is closed and the total energy $E=\sum_{p} \epsilon_{p} n_{p}$ cannot fluctuate. Accordingly, $\sum_{p} \epsilon_{p}\left\langle\Delta n_{p} \Delta n_{k}\right\rangle=0$ in this case. Note that the correlation is negative as it should be, since finiteness of $C_{A}$ suppresses fluctuations of $E$, which means that if one $n_{p}$ increases, others are more likely to decrease. This negative correlation is therefore a direct consequence of energy conservation, and should persist even in systems which are less ideal than the one we are analyzing in this Section.

The microscopic correlator (50) determines the fluctuations of many observables. For example, by convolving it with $\epsilon_{p} \epsilon_{k}$ as in (9) one can derive the result (48). This is yet another check of (50). Note also that the correlation term in (50) is down by a factor of $1 / V$ (since $C_{A, B} \sim V$ ), where $V$ is the volume of the system. This is also easy to understand: the restriction on some linear combination of $n_{p}$ 's imposed by energy conservation affects each individual $n_{p}$ little if the number of $n_{p}$ 's (i.e., the size of the system) is large. However, the contribution of this term to fluctuations of extensive or cumulative quantities is not small, as equation (48) shows. This is due to the absence of the Kronecker delta in the second term.

We now turn to the derivation of the result (50). The uncorrelated fluctuations given by formula (7) follow from the factorizable probability distribution

$$
d P\left(n_{p}\right)=\prod_{p} d n_{p} \exp \left\{-\frac{1}{2 v_{p}^{2}}\left(\Delta n_{p}\right)^{2}\right\} .
$$

\footnotetext{
${ }^{16}$ A careful reader may note that eq. (位) literally implies that $\Delta n_{p} \sim V^{0}$, as far as the thermodynamic limit $1 / V$ power counting is concerned. $n_{p}$ is also of order $V^{0}$. If it were the case that $\Delta n_{p} \sim n_{p} / \sqrt{V}$, our assumption that the fluctuations of the occupation numbers $n_{p}$ are Gaussian would be immediately justified. Instead, the fluctuations of the occupation numbers $n_{p}$ are not necessarily Gaussian. This can be cured by considering, in place of $n_{p}$, the sum of occupation numbers of a set of modes in a cell $(\Delta p)^{3}$ centered at $p$ in momentum space, where $\Delta p$ is fixed as $V \rightarrow \infty$. Since the number of modes in such a set is $(\Delta p)^{3} V=\mathcal{O}(V)$ and the modes fluctuate independently, the central limit theorem will apply and make
} 
The energy $E=\sum_{p} \epsilon_{p} n_{p}$ in such an ensemble fluctuates according to $\left\langle(\Delta E)^{2}\right\rangle=\sum_{p} v_{p}^{2} \epsilon_{p}^{2}=$ $T^{2} C_{B}$.

Now, if we bring this system into thermal contact with the system A, according to $(45,46,47)$ the probability receives an additional factor: $\exp \left[-(\Delta E)^{2} /\left(2 T^{2} C_{A}\right)\right]$. For example, if $C_{A}=0$ it becomes a delta-function, meaning that the system $\mathrm{B}$ is closed itself, and the energy cannot fluctuate. So, we write:

$$
\begin{aligned}
d P\left(n_{p}\right) & =\left(\prod_{p} d n_{p}\right) \exp \left\{-\sum_{p} \frac{1}{2 v_{p}^{2}}\left(\Delta n_{p}\right)^{2}\right\} \exp \left\{-\frac{1}{2 T^{2} C_{A}}\left(\sum_{p} \epsilon_{p} \Delta n_{p}\right)^{2}\right\} \\
& =\left(\prod_{p} d n_{p}\right) \int d \lambda \exp \left\{-\sum_{p} \frac{1}{v_{p}^{2}}\left(\Delta n_{p}\right)^{2}+\lambda \sum_{p} \epsilon_{p} \Delta n_{p}\right\} \exp \left\{T^{2} C_{A} \frac{\lambda^{2}}{2}\right\},
\end{aligned}
$$

where we have introduced a Lagrange multiplier $\lambda$. The integration over $\lambda$ should be done along the imaginary axis for convergence.

Completing the squares we find:

$$
\begin{aligned}
d P\left(n_{p}, \lambda\right)= & d \lambda\left(\prod_{p} d n_{p}\right) \exp \left\{-\sum_{p} \frac{1}{2 v_{p}^{2}}\left(\Delta n_{p}-\lambda v_{p}^{2} \epsilon_{p}\right)^{2}\right\} \\
& \times \exp \left\{\left(T^{2} C_{A}+\sum_{p} v_{p}^{2} \epsilon_{p}^{2}\right) \frac{\lambda^{2}}{2}\right\} .
\end{aligned}
$$

Now we see that:

$$
\begin{aligned}
& \left\langle\left(\Delta n_{p}-\lambda v_{p}^{2} \epsilon_{p}\right)\left(\Delta n_{k}-\lambda v_{k}^{2} \epsilon_{k}\right)\right\rangle=v_{p}^{2} \delta_{p k}, \\
& \left\langle\lambda^{2}\right\rangle=-\left(T^{2} C_{A}+\sum_{p} v_{p}^{2} \epsilon_{p}^{2}\right)^{-1}=\frac{-1}{T^{2}\left(C_{A}+C_{B}\right)},
\end{aligned}
$$

and

$$
\left\langle\lambda\left(\Delta n_{k}-\lambda v_{k}^{2} \epsilon_{k}\right)\right\rangle=0,
$$

from which we find:

$$
\begin{aligned}
\left\langle\Delta n_{p} \Delta n_{k}\right\rangle & =v_{p}^{2} \delta_{p k}+\left\langle\lambda \Delta n_{p}\right\rangle v_{k}^{2} \epsilon_{k}+\left\langle\lambda \Delta n_{k}\right\rangle v_{p}^{2} \epsilon_{p}-\left\langle\lambda^{2}\right\rangle v_{p}^{2} \epsilon_{p} v_{k}^{2} \epsilon_{k} \\
& =v_{p}^{2} \delta_{p k}+\left\langle\lambda^{2}\right\rangle v_{p}^{2} \epsilon_{p} v_{k}^{2} \epsilon_{k} \\
& =v_{p}^{2} \delta_{p k}-\frac{1}{T^{2}} \frac{v_{p}^{2} \epsilon_{p} v_{k}^{2} \epsilon_{k}}{C_{A}+C_{B}} .
\end{aligned}
$$

Q.E.D.

Now, armed with the equation (50), we can calculate all other fluctuations in our ideal Bose gas $B$ in contact with the system $A$.

fluctuations of such "smeared" $n_{p}$ Gaussian. Practically, we always convolve $n_{p}$ with a smooth function of $p$. Instead of displaying the smearing of $n_{p}$ explicitly in our notation, we can instead just treat the fluctuations of $n_{p}$ as if they are Gaussian, because this will not affect any of the quantities calculated by convolving $n_{p}$ with a smooth function of $p$. 


\subsection{Application: Fluctuations of Mean $p_{T}$}

As an example of the application of the formula for the microscopic correlator (50) we analyze the fluctuations of an intensive variable in the ideal Bose gas of pions which we denote $q$, $q=Q / N$. We wish to see how the fluctuations of $q$ are influenced by the fact that the pions are in thermal contact with a system with (possibly singular) heat capacity $C_{A}$. We shall be interested in a particular case where $q$ is the mean transverse momentum $p_{T}$, but shall use the more general notation both for later convenience and to make contact with Section 2 in which we discussed $\epsilon=E / N$, another possible $q$.

Starting from an equation similar to (17), averaging, and using the correlator (50) instead of (7) we obtain:

$$
\begin{aligned}
\left\langle(\Delta q)^{2}\right\rangle & =\left\langle\left(\Delta\left(\frac{Q}{N}\right)\right)^{2}\right\rangle \\
& =\frac{1}{\langle N\rangle^{2}}\left\{\sum_{p} v_{p}^{2}\left(q_{p}-\langle q\rangle\right)^{2}-\frac{1}{T^{2}\left(C_{A}+C_{B}\right)}\left[\sum_{p} v_{p}^{2} \epsilon_{p}\left(q_{p}-\langle q\rangle\right)\right]^{2}\right\} .
\end{aligned}
$$

The first term on the right-hand side is the same as in (22) with $q=\epsilon_{p}$. This is the main contribution to $\left\langle(\Delta q)^{2}\right\rangle$. We have seen that these thermodynamic fluctuations can be described using the variance of the inclusive single-particle distribution and the Bose enhancement factor. The second, negative, term in (58) is the effect of the anti-correlation (second term in (50)) induced by energy conservation and thermal contact with the system $A$. This term would be nonzero even if $C_{A}$ were zero. In this case, it would describe the effects of energy conservation on the fluctuations of $q$ in the system $B$. Thermal contact with the system $A$ reduces this term, but it remains important as long as $C_{A}$ is comparable to $C_{B}$. It vanishes at the point where $C_{A}$ diverges.

In a heavy ion collision, the heat capacity of the pion gas $C_{B}$ is a sizable fraction of the total heat capacity $C_{A}+C_{B}$. The effect (58) can therefore decrease the fluctuations of $p_{T}$, countering the Bose enhancement. This effect will be reduced as we approach the critical point where $C_{A}$ diverges. This will lead to an increase in the event-by-event fluctuations of $p_{T}$ as compared to the variance of the inclusive single-particle spectrum.

To make the comparison with the Bose enhancement effect estimated in Section 3.1 easier, we shall express the strength of the effect of the thermal contact in terms of the ratio, $F_{T}$ of the whole expression in curly brackets in (58) to the first term in this expression. For the fluctuation of mean transverse momentum per event, i.e., for $q=p_{T}$ we find

$$
F_{T} \approx 1-\frac{0.12}{C_{A} / C_{B}+1}
$$

for $T=120 \mathrm{MeV}$ and $\mu_{\pi}=0$. We see that the effects of energy conservation and thermal contact on the fluctuations of an intensive quantity like $p_{T}$ are smaller than the effects on the fluctuations of the energy in (48). Several obstacles make it difficult to use (59) quantitatively. First, some dilution of the effect is to be expected because less than half of the pions which are observed are direct. Second, it is a little bit difficult to know how to estimate $C_{A} / C_{B}$, because we have analyzed such an idealized situation. The system $A$ should certainly include the neutral pions in the same region of rapidity as the observed charged 
pions; however, should the pions at different rapidities be included? The total number of pions, neutral and charged, in a central event at the SPS at $160 \mathrm{AGeV}$ is about ten times larger than the number of charged pions per event used in NA49's present analysis. This suggests that $C_{A} / C_{B}$ is at the very most 10 . If we take $C_{A} / C_{B} \sim 3$ for orientation, which can be justified if one assumes that $C_{A}$ includes the heat capacity of the resonances and that of the neutral pions in the same region of phase space as the observed pions, we find $F_{T}-1$ of the order of $-3 \%$, before taking into account the dilution by non-direct pions. The effect is comparable in magnitude to the Bose enhancement, acts in the opposite direction, and should be reduced near the critical point at which $C_{A}$ diverges.

A divergent specific heat is only possible in an infinite system. In Section 5 we will estimate that in a realistic heavy ion collision, finite size effects suggest that near the critical point the sigma contribution to $C_{A}$ could be as much as a factor of $\sim 6^{2}$ larger than the contribution of a typical light degree of freedom. This suggests that $C_{A}$ could easily increase by as much as an order of magnitude at the critical point, reducing the anti-correlation in $\left\langle\Delta n_{p} \Delta n_{k}\right\rangle$ and the negative contribution to $F_{T}$ by the same factor.

The effects of thermal contact can be distinguished from other effects, like those of finite two-track resolution, which also counter the Bose enhancement effect because of the specific form of the microscopic correlator (50). The effect of energy conservation and thermal contact introduces an off-diagonal (in $p k$ space) anti-correlation. Although our estimate of the magnitude of the effect suffers from a variety of uncertainties introduced by the idealizations used throughout this Section, the existence of this off-diagonal anti-correlation is robust. It arises simply due to energy conservation: when one $n_{p}$ fluctuates up others must fluctuate downward, and it is therefore more likely that $n_{k}$ fluctuates downward. If $C_{A}$ is increased, then the system A can more easily supply the energy needed for the upward fluctuation in $n_{p}$, and the anti-correlation between $n_{p}$ and $n_{k}$ is reduced. Preliminary analysis by NA49 suggests that some amount of such anti-correlation is observed in the data[28]. It will be interesting to compare the magnitude of any effect observed in the data with our estimates. We leave this to future work. If it is possible to separate this effect from other effects because it is an off-diagonal anti-correlation, then a measurement of this effect would yield an estimate for the effective value of the ratio $C_{A} / C_{B}$ at freeze-out.

Note that $F_{T}$ increases near the critical point, but it increases towards a finite value (namely 1.) In contrast, in Section 5 we will explore effects which result in the divergence of an analogously defined ratio $F_{\sigma}$ at the critical point.

\subsection{Another Application: $\tilde{T}$}

In this subsection we introduce another measure of the temperature of the pion gas, $\tilde{T}$. Our

new variable $\tilde{T}$ is well-defined on a single event, and has the property that $\langle\tilde{T}\rangle$ is related to the slope parameter. We have found that although $\left\langle p_{T}\right\rangle$ is related to $T$, the fluctuations $\left\langle\left(\Delta p_{T}\right)^{2}\right\rangle$ are not at all like the fluctuations $\left\langle(\Delta T)^{2}\right\rangle$ in (1). We now show that $\left\langle(\Delta \tilde{T})^{2}\right\rangle$ also does not behave quite like $\left\langle(\Delta T)^{2}\right\rangle$. The reader should expect this, since we argued on general grounds that (1) can only be obtained from a mechanical observable if the equation of state of the system $A$ is known. Still, it is nice to confirm this using an example of an observable which is a less straightforward intensive quantity than just $\epsilon=E / N$ or $p_{T}$. 
We define for each member of the ensemble (i.e., for each event) independently:

$$
\chi^{2}(T)=\frac{1}{2} \sum_{p}\left(n_{p}-n_{p}^{0}(T)\right)^{2} \frac{1}{\sigma_{p}^{2}},
$$

where

$$
n_{p}^{0}(T)=\frac{1}{e^{\epsilon_{p} / T}-1},
$$

and $\sigma_{p}^{2}$ is some function of $p$ which we can choose for convenience later. Then for each event individually we can define a temperature, $\tilde{T}$, which is found by minimizing $\chi^{2}(T)$ for this event:

$$
\left[\frac{d \chi^{2}(T)}{d T}\right]_{T=\tilde{T}}=0 .
$$

It is clear that mean value of $\tilde{T}$ over all events for the ideal Bose gas will coincide with the actual temperature of the gas $T$. But, since $\tilde{T}$ is defined for a single event, rather then for the whole ensemble, it fluctuates!

As before, the fluctuations in $\tilde{T}$ are determined by fluctuations of $n_{p}$. For small fluctuation $\Delta \tilde{T}$ we can write:

$$
\begin{aligned}
0= & \Delta\left[\frac{d \chi^{2}(T)}{d T}\right]_{T=\tilde{T}}=\Delta\left[\sum_{p}\left(n_{p}^{0}(T)-n_{p}\right) \frac{\partial n_{p}^{0}}{\partial T} \frac{1}{\sigma_{p}^{2}}\right] \\
= & \sum_{p}\left(\Delta T \frac{\partial n_{p}^{0}}{\partial T}-\Delta n_{p}\right) \frac{\partial n_{p}^{0}}{\partial T} \frac{1}{\sigma_{p}^{2}},
\end{aligned}
$$

where we omit the tilde on $T$. Note that even if $\sigma_{p}^{2}$ contains dependence on $T$, it will be always multiplied by $\left(n_{p}-n_{p}^{0}\right)$ which is zero to the relevant order in the size of the fluctuation. Now we need:

$$
\frac{\partial n_{p}^{0}(T)}{\partial T}=\frac{1}{T^{2}} \epsilon_{p} n_{p}^{0}\left(1+n_{p}^{0}\right)=\frac{1}{T^{2}} \epsilon_{p} v_{p}^{2},
$$

according to (田). We rewrite (63) as:

$$
\frac{\Delta T}{T^{2}} \sum_{p} \epsilon_{p}^{2} v_{p}^{4} \frac{1}{\sigma_{p}^{2}}=\sum_{p} \Delta n_{p} \epsilon_{p} v_{p}^{2} \frac{1}{\sigma_{p}^{2}} .
$$

We can carry on with an arbitrary $\sigma_{p}^{2}$, but let us make the following choice: $\sigma_{p}^{2}=v_{p}^{2}$. This choice makes a lot of sense if one recalls that in the standard definition of $\chi^{2}$ one divides each square deviation term by its normal square deviation (which is usually obtained from experimental error, and which here we know to be $v_{p}^{2}$ from the fluctuations of $n_{p}$ ). This choice simplifies formulas.

Now we square (65), average over events, and restore the tilde on $T$ :

$$
\frac{\left\langle(\Delta \tilde{T})^{2}\right\rangle}{\tilde{T}^{4}}\left[\sum_{p} \epsilon_{p}^{2} v_{p}^{2}\right]^{2}=\sum_{p} \sum_{k} \epsilon_{p} \epsilon_{k}\left\langle\Delta n_{p} \Delta n_{k}\right\rangle,
$$


which, according to (50) means:

$$
\frac{\left\langle(\Delta \tilde{T})^{2}\right\rangle}{\tilde{T}^{2}}=\frac{1}{C_{B}} \frac{C_{A}}{C_{A}+C_{B}} .
$$

We see that fluctuations of $\tilde{T}$, like those of $p_{T}$, increase towards the critical point of the system $A$, where $C_{A} \rightarrow \infty$, approaching a finite constant. When $C_{A}$ is infinite, the system $B$ (the Bose gas) is in the canonical ensemble, and the fluctuations of $\tilde{T}$ are given precisely by (11), with the specific heat $C_{B}$ in the denominator.[7]

Different definitions of the temperature $\tilde{T}$ can be devised (using different choices of $\sigma_{p}^{2}$ ). They will lead to different temperatures for a given event, which are the same in the mean (and equal to $T$ ), but different in the size of their fluctuations. All these fluctuations will increase somewhat at the critical point, but will not diverge there as they are controlled there by $C_{B}$ (or some other property of the thermometer $B$ ) which is nonsingular.

\subsection{Two Further Applications: $\left\langle(\Delta N)^{2}\right\rangle$ and $\left\langle\Delta N \Delta p_{T}\right\rangle$}

Once we understand how some physical effect influences the microscopic correlator $\left\langle\Delta n_{p} \Delta n_{k}\right\rangle$, we can calculate the fluctuations of many different observables. The task then is to look for observables in which the effect of interest is large, and which are of practically utility in the sense that they are easily accessible to experimental analysis. We give two further simple examples here.

In Section 4.3, we analyzed the fluctuations of an intensive quantity, $p_{T}$, and obtained the expression (58) for $\left\langle\left(\Delta p_{T}\right)^{2}\right\rangle$. Similarly, we can use the microscopic correlator (50) to analyze the fluctuations of the extensive quantity $N$, and obtain

$$
\left\langle(\Delta N)^{2}\right\rangle=\sum_{p} v_{p}^{2}-\frac{1}{T^{2}\left(C_{A}+C_{B}\right)}\left[\sum_{p} v_{p}^{2} \epsilon_{p}\right]^{2} .
$$

The first term is the ideal Bose gas result, and the second term is the correction due to thermal contact and energy conservation. For $T=120 \mathrm{MeV}, \mu_{\pi}=0$, the effect of the second term is to multiply $\left\langle(\Delta N)^{2}\right\rangle$ by a factor of $\left[1-0.20 /\left(1+C_{A} / C_{B}\right)\right]$. Note, however, that the multiplicity fluctuations of the pions obtain from the resonance gas which we analyzed in Section 3 are dominated by the pions from those resonances which decay into more than one pion. Doubling the contribution of the direct pions to $\left\langle(\Delta N)^{2}\right\rangle$ in the calculation (32) only increases $\left\langle(\Delta N)^{2}\right\rangle$ by $10 \%$. The effect of thermal contact and energy conservation on the direct pions seen in (68) is therefore a very small contribution to the total $\left\langle(\Delta N)^{2}\right\rangle$ of $(32)$.

We saw at the end of Section 2 that cross correlations between intensive observables and $N$ are of interest, because they vanish in a classical ideal gas. We therefore use the microscopic correlator (50) to calculate

$$
\langle\Delta N \Delta q\rangle=\frac{1}{\langle N\rangle}\left\{\sum_{p} n_{p}^{2}\left(q_{p}-\langle q\rangle\right)-\frac{1}{T^{2}\left(C_{A}+C_{B}\right)}\left[\sum_{k} v_{k}^{2} \epsilon_{k}\right]\left[\sum_{p} v_{p}^{2} \epsilon_{p}\left(q_{p}-\langle q\rangle\right)\right]\right\} .
$$

\footnotetext{
${ }^{17}$ The heat capacity $C_{V}$ in this case is that at fixed $\mu$. It is remarkable that if we define $\tilde{T}$ by simultaneously fitting two variables in (60), $\tilde{T}$ and $\tilde{\mu}$, the resulting $\tilde{T}$ will again fluctuate according to (11), but with heat capacity at constant $N$. We leave this as an instructive exercise for our reader.
} 
For $q=p_{T}, T=120 \mathrm{MeV}, \mu_{\pi}=0$ we find

$$
\frac{\left\langle\Delta N \Delta p_{T}\right\rangle}{\left\langle p_{T}\right\rangle}=-0.021\left(1+\frac{10 .}{C_{A} / C_{B}+1}\right) \text {. }
$$

As we saw in Section 2, correlations like this arise only due to nontrivial effects, and are generally small. In this case, we see that (for $C_{A} \sim 3 C_{B}$ ) the effect of energy conservation and thermal contact is $\sim 2.5$ times as large as that due to Bose enhancement. This suggests that this correlation would be a very interesting quantity to use to look for the critical point. It is small in magnitude, but even after the dilution of the direct pions by those produced in resonance decays are taken into account, it may change by a large factor near the critical point where $C_{A} \rightarrow 0$.

In conclusion, the effects of thermal contact and energy conservation on the pions could either be found directly, by detecting the anti-correlation in the microscopic correlator $\left\langle\Delta n_{p} \Delta n_{k}\right\rangle$. Or, the resulting effects on $\left\langle\left(\Delta p_{T}\right)^{2}\right\rangle,\left\langle(\Delta \tilde{T})^{2}\right\rangle,\left\langle(\Delta N)^{2}\right\rangle$ or $\left\langle\Delta p_{T} \Delta N\right\rangle$ which we have estimated may be discovered, likely by seeing them change as control parameters are varied.

\section{Pions Near the Critical Point: Interaction with the Sigma Field}

In the previous section, we made the assumption that the "direct pions" at freeze-out could be described as an ideal Bose gas. We do not expect this to be a good approximation if the freezeout point is near the critical point. The sigma field is the order parameter for the transition and near the critical point it therefore develops large critical long wavelength fluctuations. These fluctuations are responsible for singularities in thermodynamic quantities. In the previous section, we analyzed this situation by pretending that the only effect on the pions was due to thermal contact with a heat bath with divergent susceptibilities. In this Section we take the next logical step, and consider the effect of the classical critical fluctuations on the pions through the $\sigma \pi \pi$ coupling. It would be strange if, as in the previous section, the properties of the pions remained regular in the thermodynamic limit in the presence of the nonanalytic behavior of the sigma field. We will see that the fluctuations of both the multiplicity and the mean transverse momentum of the pions do in fact diverge at the critical point.

We then estimate the size of the effects in a heavy ion collision. This requires first estimating the strength of the coupling constant $G$, and then taking into account the finite size of the system and the finite time during which the long wavelength fluctuations can develop. The pion fluctuations induced by the $G \sigma \pi \pi$ interaction are divergent and are therefore the dominant fluctuations in an infinite system. In the finite system of interest, we find that the momentum fluctuations are large enough to be easily detectable, but not so large as to seriously jeopardize our treatment, which considers the effects of the interaction only to lowest order. It is for this reason that we have first analyzed all effects other than those introduced by the $G \sigma \pi \pi$ interaction, and now add these effects in. The multiplicity fluctuations are large enough that in this case a treatment which goes beyond lowest order in $G$ seems called for. We leave this to the future. 


\subsection{Microscopic Correlator}

As before, we shall derive the expression for the microscopic "master" correlator, $\left\langle\Delta n_{p} \Delta n_{k}\right\rangle$, which can then be used to calculate fluctuations of various observables. We neglect the effects considered in the previous Section, as they can be added to the effects of this Section at the end. We concentrate on the fluctuations of the sigma field, the fluctuations of the pion occupation numbers, and the $\sigma \pi \pi$ coupling. The long wavelength fluctuations of the sigma field which are responsible for the singular effects of interest are classical.

The effective potential $\Omega$ determines the probability distribution of the classical field $\sigma$ through

$$
d P(\sigma)=d \sigma \exp \left\{-\frac{\Omega(\sigma)}{T}\right\} .
$$

This equation can actually be thought of as the definition of $\Omega(\sigma)$. The effective potential is extensive, but for convenience we set the volume $V=1$ in the calculations to follow, although we will restore it explicitly in our results. (Note that throughout previous sections, we had set $V=1$ implicitly. The momentum sum $\sum_{p}$ should always be read as $V \int d^{3} p /(2 \pi)^{3}$.) Let us consider small fluctuations of the field $\sigma$ around the minimum of $\Omega(\sigma)$. We can then expand the effective potential $\Omega(\sigma)$ around $\sigma=0$. The first terms will be

$$
\Omega(\sigma)=\frac{m_{\sigma}^{2} \sigma^{2}}{2}+G \sigma: \pi^{2}:+\mathcal{O}\left(\sigma^{3}\right),
$$

where we have temporarily omitted terms independent of $\sigma$ (such as $\left.m_{\pi}^{2} \pi^{2} / 2\right)$. The second term is the interaction between sigmas and pions. The coupling $G$ has the dimensions of mass, and its magnitude near the critical point will be estimated below. The notation :: signifies tadpole subtraction: : $\pi^{2}:=\pi^{2}-\left\langle\pi^{2}\right\rangle$, which makes sure that the minimum of $\sigma$ is not shifted as we shall see below. (The notation $\pi^{2}$ itself is itself somewhat symbolic, as it represents $\int d^{3} x \pi(x) \pi(x)$.) Thus we have:

$$
d P(\sigma)=d \sigma \exp \left\{-\frac{m_{\sigma}^{2} \sigma^{2}}{2 T}-\frac{G}{T} \sigma: \pi^{2}:\right\}
$$

Now, the field $\pi$ also fluctuates. Let us determine the corresponding (joint) probability distribution. In the previous section we used the probability distribution for the occupation numbers, and we begin by translating the fluctuations of the field $\pi$ into fluctuations of the occupation numbers. We write, doing the usual Fourier transform:

$$
\pi^{2}=\sum_{p}\left|\pi_{p}\right|^{2}
$$

We can relate the Fourier components $\pi_{p}$ to the occupation numbers $n_{p}$. It is clear that $n_{p} \sim\left|\pi_{p}\right|^{2}$. The coefficients can be determined, for example, by using

$$
Z=\int \mathcal{D} \pi \exp \left\{-\int_{0}^{1 / T} d t \int d V\left[\frac{1}{2}\left(\partial_{\mu} \pi\right)^{2}+\frac{1}{2} m_{\pi}^{2} \pi^{2}\right]\right\}=Z_{T=0} \prod_{p}\left(1-e^{-\omega_{p} / T}\right) .
$$

\footnotetext{
${ }^{18}$ Clearly, the fluctuations of $\sigma$ are not small. We shall proceed with the assumption that the higher-order terms in $\Omega(\sigma)$ yield subleading contributions to the singular effect we seek. We shall return to this point in Section 5.4. Also note that we consider only the zero momentum mode of the field $\sigma$. This can be justified in a diagrammatic approach, which can also handle nonzero momentum modes of $\sigma$.
} 
Differentiating $\ln Z$ with respect to $m_{\pi}^{2}$ we find

$$
\left\langle\pi^{2}\right\rangle=\sum_{p} \frac{1}{\omega_{p}}\left\langle n_{p}\right\rangle
$$

up to the temperature independent vacuum contribution (equal to $\sum_{p} \omega_{p} / 2$ ) from $\ln Z_{T=0}$. So we have

$$
\pi^{2}=\sum_{p} \frac{1}{\omega_{p}} n_{p}
$$

Note now that $\left\langle\pi^{2}\right\rangle=\sum_{p}\left\langle n_{p}\right\rangle / \omega_{p} \neq 0$. So, unless we subtract $\left\langle\pi^{2}\right\rangle$ the minimum of $\sigma$ will be shifted from the origin (this subtraction will also take care of the vacuum fluctuations). We have:

$$
\pi^{2}-\left\langle\pi^{2}\right\rangle=\sum_{p} \frac{\Delta n_{p}}{\omega_{p}}
$$

Now, putting everything together, we find the joint probability distribution for the sigma field and for the pion occupation numbers:

$$
d P\left(\sigma, n_{p}\right)=d \sigma\left(\prod_{p} d n_{p}\right) \exp \left\{-\sum_{p} \frac{1}{2 v_{p}^{2}}\left(\Delta n_{p}\right)^{2}-\frac{G \sigma}{T} \sum_{p} \frac{\Delta n_{p}}{\omega_{p}}-\frac{m_{\sigma}^{2}}{2 T} \sigma^{2}\right\} .
$$

This is a very important formula which will allow us to calculate the fluctuations.

The measure $d P\left(\sigma, n_{p}\right)$ is Gaussian, which is very helpful. Completing the squares, we find

$$
\begin{aligned}
d P\left(\sigma, n_{p}\right)= & d \sigma\left(\prod_{p} d n_{p}\right) \exp \left\{-\sum_{p} \frac{1}{2 v_{p}^{2}}\left(\Delta n_{p}+\frac{G \sigma}{T} \frac{v_{p}^{2}}{\omega_{p}}\right)^{2}\right\} \\
& \times \exp \left\{-\left(\frac{m_{\sigma}^{2}}{2 T}-\frac{G^{2}}{T^{2}} \sum_{p} \frac{v_{p}^{2}}{2 \omega_{p}^{2}}\right) \sigma^{2}\right\}
\end{aligned}
$$

Before we make the final and the simplest step, let us make two side notes.

The equation (80) shows that the interaction with $\sigma$ shifts mean occupation numbers by

$$
\delta\left\langle n_{p}\right\rangle=-\frac{G \sigma}{T} \frac{v_{p}^{2}}{\omega_{p}} .
$$

As the reader might have guessed already, this must be due to the shift of the mass of the pions linear in $\sigma$, which can be seen from $(\sqrt{72})$ :

$$
\frac{\delta m_{\pi}^{2}}{2}=G \sigma
$$

It is trivial to evaluate the change in $\left\langle n_{p}\right\rangle$ induced by the change of the mass. Since $\epsilon_{p}=$ $\left.\sqrt{p^{2}+m_{\pi}^{2}}-\mu\right)$ we find:

$$
\delta\left\langle n_{p}\right\rangle=-\left\langle n_{p}\right\rangle\left(1+\left\langle n_{p}\right\rangle\right) \frac{\delta \epsilon_{p}}{T}=-v_{p}^{2} \frac{1}{T} \frac{\delta m_{\pi}^{2}}{2 \omega_{p}}
$$


which is the same as (81) with (82). The fluctuations of the sigma field will have further affects on the pion occupation numbers, but these are higher order in $G$ and we neglect them here.

The second side note is more important. We see from (80) that the mass of the $\sigma$ field is corrected by the fluctuations of the pions:

$$
\tilde{m}_{\sigma}^{2}=m_{\sigma}^{2}-\frac{G^{2}}{T^{2}} \sum_{p} \frac{v_{p}^{2}}{\omega_{p}^{2}} .
$$

Diagrammatically, this corresponds to the thermal one-loop diagram $\sigma \rightarrow \pi \pi \rightarrow \sigma$. The physical mass of the sigma is $\tilde{m}_{\sigma}$, to the order in which we are working. This is the mass which vanishes at the critical point. We shall omit the tilde in the following.

Finally, we can read off the following expectation values from the probability distribution (80):

$$
\begin{aligned}
& \left\langle\left(\Delta n_{p}+\frac{G \sigma}{T} \frac{v_{p}^{2}}{\omega_{p}}\right)\left(\Delta n_{k}+\frac{G \sigma}{T} \frac{v_{k}^{2}}{\omega_{k}}\right)\right\rangle=v_{p}^{2} \delta_{p k} \\
& \left\langle\sigma^{2}\right\rangle=\frac{T}{m_{\sigma}^{2}} ; \\
& \left\langle\sigma \Delta n_{p}\right\rangle=-\left\langle\sigma^{2}\right\rangle \frac{G}{T} \frac{v_{p}^{2}}{\omega_{p}}
\end{aligned}
$$

This gives

$$
\left\langle\Delta n_{p} \Delta n_{k}\right\rangle=v_{p}^{2} \delta_{p k}+\frac{1}{m_{\sigma}^{2}} \frac{G^{2}}{T} \frac{v_{p}^{2} v_{k}^{2}}{\omega_{p} \omega_{k}} .
$$

We see that the coupling of the pions to the sigma field leads to a singular contribution to the correlator of the pion fluctuations as we approach the critical point at which $m_{\sigma}=0$. The first term on the right hand side describes the variance of the inclusive distribution and the Bose enhancement effect as we saw in Section 3. The additional terms which we discovered in Section 4 could now be added to the right hand side. It is of course the new, divergent term on which we shall focus our attention.

One can represent both terms in this equation diagrammatically as in Fig. 2. The singular term is due to the exchange of the sigma in the process of forward pion-pion scattering. This results in a characteristic $1 / m_{\sigma}^{2}$ singularity. A different way of deriving the formula for the correlator would be to do a straightforward diagrammatic expansion of $\left\langle\Delta n_{p} \Delta n_{k}\right\rangle$. This will also allow one to include the effects of the nonzero momentum modes of the sigma field. The second term in (88) is the most singular one in this correlator as $m_{\sigma} \rightarrow 0$ because it involves the exchange of the sigma field with zero momentum. We defer an analysis of less singular terms using the diagrammatic approach to future work. 


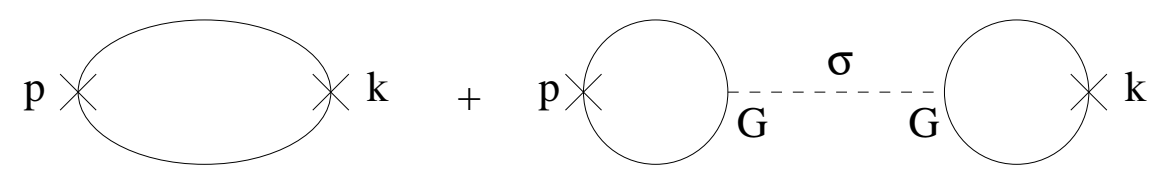

Figure 2: Diagrammatic representation of the right hand side of the correlator (88). The crosses represent the insertions of $\Delta n_{p}=: \pi_{p} \pi_{p}^{*}:$. The solid/dashed lines are the pion/sigma field thermal propagators.

\subsection{Application: Fluctuations of Mean $p_{T}$}

Using (88), we can determine the fluctuations of any thermodynamic observable which can be expressed in terms of the pion occupation numbers. For a generic intensive observable $q=Q / N$ we find

$$
\begin{aligned}
\left\langle(\Delta q)^{2}\right\rangle & =\left\langle\left[\Delta\left(\frac{Q}{N}\right)\right]^{2}\right\rangle \\
& =\frac{1}{\langle N\rangle^{2}}\left\{\sum_{p} v_{p}^{2}\left(q_{p}-\langle q\rangle\right)^{2}+\frac{1}{V m_{\sigma}^{2}} \frac{G^{2}}{T}\left[\sum_{p} \frac{v_{p}^{2}}{\omega_{p}}\left(q_{p}-\langle q\rangle\right)\right]^{2}\right\} .
\end{aligned}
$$

where we have displayed the factor of $V$ explicitly to show that both terms are of the same order in $V$. (Recall that $\sum_{p} \rightarrow V \int d^{3} p /(2 \pi)^{3}$.) As before, the intensive observable of primary interest will be the mean transverse momentum in an event, $q=p_{T}$. It is clear from (89) that the fluctuations of $p_{T}$ increase near the critical point and diverge at the critical point, where $m_{\sigma}$ vanishes. We will give a quantitative estimate of the effect in Section 5.5. We must first estimate the size of the coupling $G$ and the value of $m_{\sigma}$ near the critical point.⿴囗十

\subsection{The Size of the Coupling $G$}

The strength of the singular contribution to the pion correlator near the critical point depends on the size of the coupling $G$ between the $\sigma$ and $\pi$ :

$$
\mathcal{L}_{I}=G \sigma \pi_{i} \pi_{i}
$$

where the isospin index $i=1,2,3$ is summed. We first make a phenomenological estimate of the magnitude of $G$ in vacuum, and then estimate by how much $G$ is reduced near the critical point E.

The value of this coupling in the vacuum can be inferred independently from two considerations: (i) from the relationship between the sigma and pion masses and $f_{\pi}$; (ii) more directly, from the width of the sigma. We shall use both and compare.

One way of estimating the vacuum value of $G$ is to use the Gell-Mann - Lévy linear sigma model 29], in which the Lagrangian describing the dynamics of the four component field $\phi_{\alpha}=\left(\phi_{0}, \boldsymbol{\pi}\right)$ is given by

$$
\mathcal{L}=\int d^{4} x\left\{\frac{1}{2} \partial^{\mu} \phi_{\alpha} \partial_{\mu} \phi_{\alpha}-\frac{\lambda}{4}\left(\phi_{\alpha} \phi_{\alpha}-v^{2}\right)^{2}+H \phi_{0}\right\},
$$

\footnotetext{
${ }^{19}$ Similarly, one can also calculate the fluctuations of $\tilde{T}$ defined in Section 4.4 and show that these fluctuations also increase near the critical point and diverge at the critical point.
} 
where the $\mathrm{O}(4)$-breaking field $H$ is proportional to the quark mass: $H=m\langle\bar{\psi} \psi\rangle / f_{\pi}$. The vacuum expectation value $\left\langle\phi_{0}\right\rangle$ is nonzero and should be set equal to $f_{\pi}$. The $\sigma$ field is then defined by $\sigma=\phi_{0}-\left\langle\phi_{0}\right\rangle$. Setting $f_{\pi}=93 \mathrm{MeV}, m_{\pi}=135 \mathrm{MeV}$ and $m_{\sigma}=600 \mathrm{MeV}$ fixes all three parameters in the potential, and in particular yields $\lambda=20.0$. If we rewrite (91) in terms of $\sigma$, we find a term $\lambda f_{\pi} \sigma \boldsymbol{\pi}^{2}$, from which we conclude that

$$
G=\lambda f_{\pi} \sim 1900 \mathrm{MeV} .
$$

This value for $G$ seems large at first sight, but such a large coupling is in fact required by experiment. In order to see this, we evaluate the width of the sigma due to its tree-level decay into two pions, and find

$$
\Gamma=\frac{3 G^{2}}{8 \pi} \frac{p}{m_{\sigma}^{2}}=\frac{3 G^{2}}{8 \pi m_{\sigma}^{2}} \sqrt{\left(\frac{m_{\sigma}}{2}\right)^{2}-m_{\pi}^{2}} \sim 300 \mathrm{MeV},
$$

where we have used $m_{\sigma}=600 \mathrm{MeV}$ as above. The width of the sigma is known experimentally to be so large that this "particle" is only seen as a broad bump in the $s$-wave $\pi$ - $\pi$ scattering cross-section. An estimate of $300 \mathrm{MeV}$ for this width is therefore reasonable. We conclude that the vacuum $\sigma \pi \pi$ coupling must be at least as large as $G \sim 1900 \mathrm{MeV}$, since the sigma would otherwise be too narrow.

Our estimate makes it clear that the vacuum value of $G$ would not change much if one were to take the chiral limit $m \rightarrow 0$. The situation is different at the critical point. Taking the quark mass to zero while following the critical endpoint leads one to the tricritical point $\mathrm{P}$ in the phase diagram for QCD with two massless quarks. At this point, $G$ vanishes as we discuss below. This suggests that at E, the coupling $G$ is less than in vacuum. Our goal in the remainder of this subsection is to use what we know about physics near the tricritical point $\mathrm{P}$ to make an estimate of how much the coupling $G$ is reduced at the critical endpoint $\mathrm{E}$ (with the quark mass $m$ having its physical value), relative to the vacuum value $G \sim 1900$ $\mathrm{MeV}$ estimated above.

We begin by recalling some known results. (For details, see Refs. [2, 3, 11.) In QCD with two massless quarks, a spontaneously broken chiral symmetry is restored at finite temperature. This transition is likely second order and belongs in the universality class of $O(4)$ magnets in three dimensions. At zero $T$, various models suggest that the chiral symmetry restoration transition at finite $\mu$ is first order. Assuming that this is the case, one can easily argue that there must be a tricritical point $\mathrm{P}$ in the $T \mu$ phase diagram, where the transition changes from first order (at higher $\mu$ than $\mathrm{P}$ ) to second order (at lower $\mu$ ), and such a tricritical point has been found in a variety of models. [2, 3, 30] The nature of this point can be understood by considering the Landau-Ginzburg effective potential for $\phi_{\alpha}$, order parameter of chiral symmetry breaking:

$$
\Omega\left(\phi_{\alpha}\right)=\frac{a}{2} \phi_{\alpha} \phi_{\alpha}+\frac{b}{4}\left(\phi_{\alpha} \phi_{\alpha}\right)^{2}+\frac{c}{6}\left(\phi_{\alpha} \phi_{\alpha}\right)^{3}
$$

The coefficients $a, b$ and $c>0$ are functions of $\mu$ and $T$. The second order phase transition line described by $a=0$ at $b>0$ becomes first order when $b$ changes sign, and the tricritical point $\mathrm{P}$ is therefore the point at which $a=b=0$. The critical properties of this point can be inferred from universality 2, 3], and the exponents are as in the mean field theory (94). We 
will use this below. Most important in the present context is the fact that because $\langle\phi\rangle=0$ at $\mathrm{P}$, there is no $\sigma \pi \pi$ coupling, and $G=0$ there.

In real QCD with nonzero quark masses, the second order phase transition becomes a smooth crossover and the tricritical point $\mathrm{P}$ becomes $\mathrm{E}$, the second order critical endpoint of a first order phase transition line. Whereas at $\mathrm{P}$ there are four massless scalar fields undergoing critical long wavelength fluctuations, the $\sigma$ is the only field which becomes massless at the point $\mathrm{E}$, and the point $\mathrm{E}$ is therefore in the Ising universality class [2, 3]. The pions remain massive at $\mathrm{E}$ because of the explicit chiral symmetry breaking introduced by the quark mass $m$. Thus, when we discuss physics near $\mathrm{E}$ as a function of $\mu$ and $T$, but at fixed $m$, we will use universal scaling relations with exponents from the three dimensional Ising model. Our present purpose, however, is to imagine varying $m$ while changing $T$ and $\mu$ in such a way as to stay at the critical point E, and ask how large $G$ (and $m_{\pi}$ ) become once $m$ is increased from zero (the tricritical point $\mathrm{P}$ at which $G=m_{\pi}=0$ ) to its physical value. For this task, we use exponents describing universal physics near P. Applying tricritical scaling relations all the way up to a quark mass which is large enough that $m_{\pi}$ is not small compared to $T_{c}$ may introduce some uncertainty into our estimate.

In order to determine the trajectory of the critical line of Ising critical points $\mathrm{E}$ as a function of quark mass $m, 0$ it is sufficient to consider the effective potential only as a function of the single component $\phi_{0} \equiv \phi$ of the four-component order parameter. When the quark mass is nonzero we can add terms containing odd powers of $\phi: \phi^{3}$ and $\phi^{5}$, in addition to just $\phi$. We shall assume that the linear term provides the leading effect, and check this assumption for self-consistency a posteriori. So, we have at nonzero $m$

$$
\Omega(\phi)=-m \phi+\frac{a}{2} \phi^{2}+\frac{b}{4} \phi^{4}+\frac{c}{6} \phi^{6} .
$$

We assume that the units of mass are chosen in such a way that the coefficient of the linear term in (95) assumes this simple form. That is, instead of writing it as $H=m M^{2}$, we are using units with $M=1$. Stable or metastable thermodynamic phases are described by minima of $\Omega$, at which $\Omega^{\prime}=0$. At the critical point $\mathrm{E}, \Omega^{\prime}=0$ and in addition both $\Omega^{\prime \prime}$ and $\Omega^{\prime \prime \prime}$ vanish. This is because three roots of the polynomial $\Omega^{\prime}(\phi)$ coalesce (two minima of $\Omega$ and one maximum in between). So, we have three conditions:

$$
\begin{aligned}
& \Omega^{\prime}=-m+a\langle\phi\rangle+b\langle\phi\rangle^{3}+c\langle\phi\rangle^{5}=0 \\
& \Omega^{\prime \prime}=a+3 b\langle\phi\rangle^{2}+5 c\langle\phi\rangle^{4}=0 \\
& \Omega^{\prime \prime \prime}=+6 b\langle\phi\rangle+20 c\langle\phi\rangle^{3}=0 .
\end{aligned}
$$

These conditions allow us to express $a, b$ and $\langle\phi\rangle$ (the value of $\phi$ at the minimum), as functions of $m$ and $c$. We neglect any change in $c$; it is the vanishing of $m$ at $\mathrm{P}$ which is of interest to us. Solving these equations by working up from the last to the first and keeping only the exponents (neglecting pre-factors) we find:

$$
a \sim m^{4 / 5} ; \quad-b \sim m^{2 / 5} ; \quad\langle\phi\rangle \sim m^{1 / 5} .
$$

${ }^{20}$ See Ref. [31] for a derivation of the analogous line of Ising points emerging from the tricritical point in the QCD phase diagram at zero $\mu$ as a function of $m$ and the strange quark mass $m_{s}$. This tricritical point can be related to the one we are discussing by varying $m_{s}[\mathbb{1}]$. 
The power $1 / 5$ is easy to understand: it is $1 / \delta$, where $\delta=5$ for the $\phi^{6}$ potential.

At $m=0$, the tricritical point $\mathrm{P}$ at $a=b=0$ has $\langle\phi\rangle=0$; the expressions (99) describe how the location of the critical point $\mathrm{E}$ in the $a b$ plane, and the value of $\langle\phi\rangle$ at $\mathrm{E}$, change as $m$ is increased from zero. From these, we will determine how $m_{\pi}$ and $G$ at $\mathrm{E}$ vary with $m$, after two asides. First, note that from these universal arguments we learn nothing about the location of the tricritical point $a=b=0$ in the $T \mu$ plane. One can only make rather crude estimates of the position of this point, as we have done in Ref. [四]. Our main purpose here and in [1] is to tell experimentalists how to find $\mathrm{P}$, so that they can find it and tell us where it is. Second, we must estimate the size of the $\phi^{3}$ and $\phi^{5}$ terms we have neglected. Assuming that both terms come with coefficients which are at least linear in $m$ (higher odd powers of $m$ are possible, but will make the size of these terms even smaller) and using the $m$ power counting of (99), we see that while all the terms in (95) are $\mathcal{O}\left(m^{6 / 5}\right)$, the $\phi^{3}$ and $\phi^{5}$ terms contribute at most $\mathcal{O}\left(\mathrm{m}^{8 / 5}\right)$ and $\mathcal{O}\left(\mathrm{m}^{2}\right)$ respectively.

To follow $m_{\pi}$ and $G$, we need the full dependence of $\Omega$ on the $\phi_{0}$ and $\boldsymbol{\pi}$ fields:

$$
\Omega(\phi, \boldsymbol{\pi})=-m \phi+\frac{a}{2}\left(\phi^{2}+\boldsymbol{\pi}^{2}\right)+\frac{b}{4}\left(\phi^{2}+\boldsymbol{\pi}^{2}\right)^{2}+\frac{c}{6}\left(\phi^{2}+\boldsymbol{\pi}^{2}\right)^{3} .
$$

For the pion mass, we need to expand around $\phi=\langle\phi\rangle$ and $\boldsymbol{\pi}=0$ and collect order $\boldsymbol{\pi}^{2}$ terms:

$$
\Omega=\frac{\pi^{2}}{2}\left(a+b\langle\phi\rangle^{2}+c\langle\phi\rangle^{4}\right)+\ldots .
$$

We can now read off the pion mass:

$$
m_{\pi}^{2}=a+b\langle\phi\rangle^{2}+c\langle\phi\rangle^{4}
$$

which, according to (96), means: $m_{\pi}^{2}=m /\langle\phi\rangle$. Using (99) we find:

$$
m_{\pi}^{2} \sim m^{4 / 5}
$$

Assuming that the dimensionful factor in this formula is of the same order of magnitude as the one in the zero $T$ and $\mu$ formula $m_{\pi}^{2} \sim m$ we conclude that the pion mass does not change much from its vacuum value, and is likely to be very slightly bigger (by a factor of order $\left.\left(\Lambda_{\mathrm{QCD}} / m\right)^{1 / 10}\right)$. This is similar to what is known to happen near $T_{c}$ for $\mu=0$. (See Ref. 31] for a review.)

To determine the constant $G$, we need to collect the $\sigma \pi^{2}$ terms in (100) where, as before, $\sigma=\phi-\langle\phi\rangle$. Only the last two terms contribute and we find

$$
\Omega=\sigma \boldsymbol{\pi}^{2}\left(b\langle\phi\rangle+2 c\langle\phi\rangle^{3}\right)+\ldots .
$$

This means $G=b\langle\phi\rangle+2 c\langle\phi\rangle^{3}$ which, according to (98), gives $G=2 b\langle\phi\rangle / 5=-4 c\langle\phi\rangle^{3} / 3$. Using the $m$ power counting (99) we find

$$
G \sim m^{3 / 5}
$$

Thus the coupling $G$ is suppressed compared to its "natural" vacuum value $G_{\text {vac }}$ by a factor of order $\left(m / \Lambda_{\mathrm{QCD}}\right)^{3 / 5}$. Taking $\Lambda_{\mathrm{QCD}} \sim 200 \mathrm{MeV}, m \sim 10 \mathrm{MeV}$ we obtain our estimate

$$
G_{E} \sim \frac{G_{\mathrm{vac}}}{6} \sim 300 \mathrm{MeV} .
$$


The main source of uncertainty in this estimate is our inability to compute the various nonuniversal masses which enter the estimate as prefactors in front of the $m$ dependence which we have followed. In other words, we do not know the correct value to use for $\Lambda_{\mathrm{QCD}}$ in the suppression factor which we write as $\left(m / \Lambda_{\mathrm{QCD}}\right)^{3 / 5}$.

\subsection{Finite Size and Finite Time Effects}

The final ingredient needed for the estimate of the size of the effect described in Sections 5.1 and 5.2 is an estimate of $m_{\sigma}$. We found that $\left\langle\Delta n_{p} \Delta n_{k}\right\rangle$ is infinite when $m_{\sigma}=0$, at E. This singularity occurs because the correlation length $\xi$ of the sigma field is infinite. In practice, however, there are important restrictions on how large $\xi$ can become. The fireball created in a heavy ion collision has a finite size and lives for a finite time; both restrict $\xi$. Similar considerations affect the estimate of the size of the effect described in Section 4.2. There, we found an anti-correlation in $\left\langle\Delta n_{p} \Delta n_{k}\right\rangle$ which vanishes as the specific heat of the system diverges. The limit on $\xi$ introduced by finite size and finite time effects also limits how large the heat capacity $C_{V}$ becomes.

We discuss finite size scaling first. If the system is infinite, a singular thermodynamic quantity such as $C_{V}$ diverges at the critical point. If the system is large relative to microscopic scales $\left(\sim 1 \mathrm{fm}\right.$ in our case) but finite, then $C_{V}$ exhibits a peak at the critical point which becomes narrower and higher as larger and larger systems are considered. Finite size scaling analysis tells us how the magnitude of the peak scales with the system size. The scaling postulate tells us that the singular parts of all observables are due to the diverging correlation length $\xi$ and can be characterized by an appropriate critical index: $Q_{\text {sing }} \sim \xi^{\Delta_{Q}}$, where $Q$ could be $C_{V}$ or some other quantity which diverges at $\mathrm{E}$. In a finite system the growth of the correlation length is limited by the size of the system: $\xi_{\max } \sim R$. Therefore, the magnitude of the singularity in a given thermodynamic quantity (the height of the peak) depends on the size of the system as

$$
Q_{\max } \sim R^{\Delta_{Q}} .
$$

Similarly, if the system is not allowed enough time to equilibrate, the singularity is also smeared. The magnitude of the singularity in this case can be estimated using finite time, or dynamic, scaling [32]. In this case the scaling postulate tells us that the typical equilibration time diverges at the critical point (critical slowing down), with this divergence related to that of the correlation length by $t \sim \xi^{\Delta_{t}}$. Reversing this relation, we conclude that if the typical time allowed for the system to equilibrate is limited to $\tau$, the correlation length can only grow up to $\xi_{\max } \sim \tau^{1 / \Delta_{t}}$. Thus, in this case

$$
Q_{\max } \sim \tau^{\Delta_{Q} / \Delta_{t}}
$$

The calculation of the numerical prefactors in (107) and (108) requires precise knowledge of the QCD dynamics and is not feasible at this time. The exponents, however, are universal and can be understood by relating them to suitable exponents in the three-dimensional Ising model. For example, the exponent for the specific heat $C_{V}$ at the end point $\mathrm{E}$ was determined in [1]:

$$
\Delta_{C_{V}}=\left(\frac{\gamma}{\nu}\right)_{3 \mathrm{~d}-\text { Ising }} \approx 2
$$


$\Delta_{C_{V}}$ is not given by the (smaller) exponent $(\alpha / \nu)_{3 \mathrm{~d} \text {-Ising }}$ because of the obliqueness of the first order phase transition line relative to the $T$ axis on the phase diagram as explained in [1]. The idea is that at the critical point, $C_{V}=\partial^{2} \Omega / \partial T^{2}$ is related to some linear combination of the Ising model susceptibilities $\partial^{2} \Omega / \partial t^{2}, \partial^{2} \Omega / \partial t \partial h$, and $\partial^{2} \Omega / \partial h^{2}$ where the Ising model temperature axis $t$ and magnetic field axis $h$ are oblique relative to the $T$ and $\mu$ axes. $C_{V}$ is controlled by the most divergent of the three Ising model susceptibilities, which is $\partial^{2} \Omega / \partial h^{2}$, and (109) results.

The dynamic scaling exponent $\Delta_{t}$, which is often called $z$, is also universal. The dynamic universality class of a system is sensitive to details of the dynamics such as whether the order parameter is or is not conserved and whether the system has other conserved quantities. The determination of $\Delta_{t}$ is a rather involved problem in some cases [32]. If we assume that QCD at the critical point E falls into the dynamic universality class of the gas-liquid phase transition (model $\mathrm{H}$ in the classification of Hohenberg and Halperin 32]) the exponent $\Delta_{t}$ can be estimated as $\Delta_{t} \approx 3$. It may therefore turn out that because $\Delta_{t}>1$ the finite time scaling restriction (108) may be somewhat more restrictive in a heavy ion collision than the finite size scaling restriction (107).

Let us estimate some typical numbers for central $\mathrm{PbPb}$ collisions at the SPS. We start with an estimate for the relevant size in the longitudinal and transverse directions beyond which $\xi$ cannot grow. The longitudinal expansion extends the longitudinal size of the fireball considerably, but regions with different rapidities freeze out at different times, and a homogeneous freeze-out at a single freeze-out time for all rapidities is not a good approximation. A similar (although not identical) problem has already been faced in two pion interferometry, which provides sizes (and durations) not of the whole system, but of a "patch" large enough that particles emitted from it can still interfere. The size of such patches depends somewhat on the direction and magnitude of the total momentum of the pion pair used in the interferometric measurement, but is an approximate measure of the size of the system over which freeze-out is homogeneous. The longitudinal size of such a patch for central $\mathrm{PbPb}$ collisions at $160 \mathrm{AGeV}$ is estimated to be[25] $2 R_{L} \approx 12-14 \mathrm{fm}$. At its ends, the rapidity difference is already about 1 . We therefore estimate that sigma field correlation length is limited by finite size effects to be less than $2 R_{L}$.

The size $D_{T}$ in the transverse direction beyond which $\xi$ cannot grow can be estimated in two ways. The initial size is that of the diameter of the nuclei, $D_{\mathrm{Pb}} \sim 14 \mathrm{fm}$. The transverse (radial) flow makes the physical size of the freeze-out surface larger than the nuclear radius, by $30-50 \%$ at freeze-out. Therefore, it must be the case that $D_{T}<20 \mathrm{fm}$. This is, however, an overestimate. Because of the relativistic transverse expansion, regions with different positions in the transverse directions cross the transition region and then freeze-out at different times. Therefore, as for $R_{L}$ above we can use the size of "patches" observed via two particle interferometry as a guide, the sigma field correlation length in the transverse directions to be less than $D_{T}=2 R_{T} \approx 10-12 \mathrm{fm}[25]$. It therefore seems that the relevant longitudinal and transverse length scales at freeze-out are about the same, and we conclude that based on finite size restrictions alone

$$
\frac{\xi}{l}<12
$$

where $l$ is the "microscopic length" of order $1 \mathrm{fm}$. 
We now turn to the restriction on the correlation length which arises from the fact that the matter created in a heavy ion system does not enjoy an infinite period of time in which to equilibrate. The expansion time can be defined through the corresponding "Hubble constant"

$$
H=\frac{1}{\tau_{H}}=\frac{d s}{s d t}
$$

where $s$ is entropy density. We use the entropy density in the definition because the total entropy is conserved during adiabatic expansion, and we are assuming that by the time the system is traversing the transition region and then freezing out, the expansion can be treated as adiabatic. Hydrodynamic models 20 suggest that at SPS energies, heavy ion collisions have $\tau_{H} \approx 10-20 \mathrm{fm} / c$. If we simply use this value of $\tau_{H}$ and neglect dimensionless factors in the scaling relation (108) we would find

$$
\frac{\xi}{l}<\left(\frac{\tau_{H}}{l}\right)^{1 / \Delta_{t}} \sim 2.5 \text {. }
$$

In spite of the long expansion time, the relatively large value of the dynamical exponent $\Delta_{t}$ can make the finite time restriction more severe than the finite size one (110). In other words, although the size of the system allows the correlation length to become as large as $12 \mathrm{fm}$, there may not be enough time for such long wavelength fluctuations to develop due to critical slowing down. The estimate (112) is suspect for several reasons. First, there may be a large dimensionless proportionality constant in this relation which is unknown to us. (In contrast, the finite size estimate (110) is a consequence of geometry, and unless the homogeneous region is larger than we estimate, it is unlikely that the finite size bound on $\xi$ can be evaded.) Second, in making the estimate (112) we have estimated the "bad" effect of critical slowing down, namely the fact that a $12 \mathrm{fm}$ correlation length will take longer than $12 \mathrm{fm}$ to develop, but we have not taken into account a compensating "good" effect of critical slowing down: because of the large specific heat, the system will spend an unusually long time with a temperature in the vicinity of the critical point. Because of the uncertainties in (112), we shall use $\xi_{\max } \sim 6 \mathrm{fm}$ as a rough estimate of the largest correlation length possible if control parameters are chosen in such a way that the system freezes out close to the critical point. More detailed study of the time evolution of the temperature of the system, of the appropriate choice for $\tau_{H}$, and of the dimensionless factors in (112) are required in order to properly estimate whether finite time effects restrict $\xi_{\max }$ further.

Since the thermal contact effect discussed in Section 4 depends on the divergent heat capacity $C_{A}$, we need to estimate how large $C_{A}$ can get, given the finiteness of the system. Using the exponent (109), we can estimate the ratio of the maximum value of that part of $C_{A}$ which would be singular in an infinite system to the "normal" value of $C_{A}$ for a degree of freedom with a correlation length $l \sim 1 \mathrm{fm}$ as

$$
\frac{\left(C_{A}\right)_{\max }^{\sigma}}{\left(C_{A}\right)_{\text {norm }}} \sim\left(\frac{\xi_{\max }}{l}\right)^{\Delta_{C_{V}}} \sim 36 .
$$

This does not mean that the specific heat $C_{A}$ is multiplied by 36 , because it receives a nonsingular contribution from other degrees of freedom. However, it suggests that in using 
Eq. (59) to estimate how much the anti-correlation induced reduction of $F_{T}$ is weakened at the critical point, it is reasonable to expect that $C_{A}$ can be up to an order of magnitude larger there than it is near $T_{c}$ far from the critical point.

We now return to our discussion of the effects of the long wavelength sigma fluctuations on the fluctuations of the pions, encoded in the microscopic correlator (88). We derived (88) using mean field theory, and would now like to discuss the effect of non-mean-field corrections. We mentioned previously that fluctuations of the sigma field around the minimum of $\Omega(\sigma)$ are not small; we now argue that this does not make much difference to the quantity of interest. One way to see how these corrections can appear is to realize that, at higher order in $G$, diagrams with $\sigma$-bubbles which are actually logarithmically divergent as $m_{\sigma} \rightarrow 0$ will contribute. These bubbles have to be resummed and may modify the exponent of the $m_{\sigma}^{-2}$ singularity in (88). This exponent is easy to infer from universality arguments.

Diagrammatically, the $1 / m_{\sigma}^{2}$ is the zero momentum value of the sigma propagator, i.e., the sigma field susceptibility. For the 3d-Ising universality class we know the corresponding exponent to be $\gamma / \nu=2-\eta$ which is $\approx 2$ to within a few percent because $\eta$ is small. We can therefore safely use the mean-field formula (88) with its $m_{\sigma}^{-2}$ divergence for our estimate, and will take $m_{\sigma} \sim 1 / \xi_{\max } \sim 1 /(6 \mathrm{fm})$. It therefore turns out that even though the effects of Section 4 depend on $C_{V}$ and the effects of this Section depend on the sigma susceptibility, both are controlled by the exponent $\gamma / \nu \approx 2$.

\subsection{Magnitude of the Effects}

We now have all the ingredients in place for our estimate of the size of the effect of the critical fluctuations of the sigma field on the fluctuations of the observed pions, via the coupling $G$. We reproduce here Eq. (89)

$$
\begin{aligned}
\left\langle(\Delta q)^{2}\right\rangle & =\left\langle\left[\Delta\left(\frac{Q}{N}\right)\right]^{2}\right\rangle \\
& =\frac{1}{\langle N\rangle^{2}}\left\{\sum_{p} v_{p}^{2}\left(q_{p}-\langle q\rangle\right)^{2}+\frac{1}{V m_{\sigma}^{2}} \frac{G^{2}}{T}\left[\sum_{p} \frac{v_{p}^{2}}{\omega_{p}}\left(q_{p}-\langle q\rangle\right)\right]^{2}\right\},
\end{aligned}
$$

which we now apply for $q=p_{T}$. We have restored the factor of $V$. The first term in the curly brackets includes the single particle inclusive distribution enhanced by the Bose effect. The second term is the effect we are interested in now. As we did in our estimate of the effects of energy conservation and thermal contact in Section 4.3, we shall express the size of the effect of interest as the ratio of the entire expression in curly brackets in (114) to the first term in these brackets. We find

$$
F_{\sigma}=1+0.35\left(\frac{G_{\text {freeze}- \text { out }}}{300 \mathrm{MeV}}\right)^{2}\left(\frac{\xi_{\text {freeze-out }}}{6 \mathrm{fm}}\right)^{2} \quad \text { for } \mu_{\pi}=60 \mathrm{MeV}
$$

and

$$
F_{\sigma}=1+0.14\left(\frac{G_{\text {freeze-out }}}{300 \mathrm{MeV}}\right)^{2}\left(\frac{\xi_{\text {freeze-out }}}{6 \mathrm{fm}}\right)^{2} \quad \text { for } \mu_{\pi}=0
$$


where we have taken $T=120 \mathrm{MeV}$. As in Section 4, the effect will be diluted by about a factor of two because not all of the pions which are observed are direct. We have written the estimates (115, 116) in such a way that the largest uncertainties are manifest. The size of the effect depends quadratically on the coupling $G$. We argued above that $G$ is reduced to $G_{E} \sim 300 \mathrm{MeV}$ at the critical point but, as we explained, there are caveats in this argument. Furthermore, freeze-out may occur somewhat away from the critical point, in which case $G$ would be somewhat larger, although still much smaller than its vacuum value. The size of the effect also depends quadratically on the sigma correlation length at freeze-out, and we have seen that there are many caveats in an estimate like $\xi_{\text {freeze-out }} \sim \xi_{\max } \sim 6 \mathrm{fm}$. Finally, the effect is sensitive to the value of $\mu_{\pi}$. There are reasons to believe that $\mu_{\pi}$ may be smaller near the critical point than far from it. Recall that $\mu_{\pi}$ is zero at chemical freeze-out, and then grows until thermal freeze-out. At the critical point, the transition temperature $T_{c}$ is somewhat lower than at lower baryon chemical potential $\mu$, and this suggests that $T_{\text {ch }}$ may be lower than the value measured in $160 \mathrm{AGeV}$ collisions. Furthermore, we have argued in Ref. [1] that the thermal freeze-out temperature $T_{\mathrm{f}}$ will be somewhat higher in the vicinity of the critical point, because the system lingers there and expands for a while with a temperature near $T_{c}{ }^{2}$ If the temperature window $T_{\mathrm{ch}}-T_{\mathrm{f}}$ is small near the critical point, then $\mu_{\pi}$ may be significantly smaller than $60 \mathrm{MeV}$ there.

We have studied two different effects on $\sqrt{F}$ in Sections 4 and 5 . The effects of energy conservation and thermal contact lead to a few percent reduction in $\sqrt{F}$; this reduction will be significantly lessened near the critical point due to the divergence of $C_{A}$. This yields perhaps a $1-2 \%$ increase in $\sqrt{F}$ near the critical point. The direct effect of the critical fluctuations which we have estimated in this section is a further, larger, increase in $\sqrt{F}$ by a factor of $\sqrt{F_{\sigma}}$. We have displayed the various uncertainties in the factors contributing to our estimates (115, 116) so that when an experimental detection of an increase and then subsequent decrease in $\sqrt{F}$ occurs, as control parameters are varied and the critical point is approached and then passed, we will be able to use the measured magnitude of this nonmonotonic effect to constrain these uncertainties. It should already be clear that an effect as large as $10 \%$ in $\sqrt{F_{\sigma}}$ is easily possible; this would be 50 times larger than the statistical error in the present data.

Once we have evaluated the microscopic correlator $\left\langle\Delta n_{p} \Delta n_{k}\right\rangle$, we can estimate the effect of the critical fluctuations of the sigma field on the fluctuations of many different pion observables. To this point, we have focussed on $\left\langle\left(\Delta p_{T}\right)^{2}\right\rangle$; we now give a brief account of the effect on $\left\langle(\Delta N)^{2}\right\rangle$ and $\left\langle\Delta N \Delta p_{T}\right\rangle$. We can use the microscopic correlator (88) to obtain

$$
\begin{aligned}
\left\langle(\Delta N)^{2}\right\rangle & =\sum_{p} v_{p}^{2}+\frac{1}{V m_{\sigma}^{2}} \frac{G^{2}}{T}\left[\sum_{p} \frac{v_{p}^{2}}{\omega_{p}}\right]^{2} \\
& \approx \sum_{p} v_{p}^{2}\left\{1+1.0\left(\frac{G_{\text {freeze-out }}}{300 \mathrm{MeV}}\right)^{2}\left(\frac{\xi_{\text {freeze-out }}}{6 \mathrm{fm}}\right)^{2}\right\},
\end{aligned}
$$

for $q=p_{T}, T=120 \mathrm{MeV}$ and $\mu_{\pi}=0$. The coefficient which is 1.0 in the last line of (117) increases to 2.0 if $\mu_{\pi}=60 \mathrm{MeV}$. We see that there can easily be a very large increase in

\footnotetext{
${ }^{21}$ The expansion rate $H$ of (111) does not decrease. However, the rate of change of $T$ with time is reduced because of the large specific heat.
} 
the multiplicity fluctuations of the direct pions near the critical point, due to the coupling between the direct pions and the critical fluctuations of the sigma field. As we have noted previously, the noncritical fluctuations of the total pion multiplicity are dominated by the pions from resonance decay. Using the result (32), we estimate that the sigma induced critical multiplicity fluctuations of the direct pions can easily lead to a $10 \%$ increase in the total $\left\langle(\Delta N)^{2}\right\rangle$. This is comparable in magnitude to the effect on $\left\langle\left(\Delta p_{T}\right)^{2}\right\rangle$, and should be easily detectable. We will see in Section 6 that there should be even a further increase in the fluctuations of the multiplicity of those pions with low $p_{T}$.

Turning now to the cross correlation between an intensive quantity and $N$, we use the microscopic correlator (88) to calculate

$$
\begin{aligned}
\frac{\langle\Delta N \Delta q\rangle}{\langle q\rangle} & =\frac{1}{\langle N\rangle\langle q\rangle}\left\{\sum_{p} n_{p}^{2}\left(q_{p}-\langle q\rangle\right)+\frac{1}{V m_{\sigma}^{2}} \frac{G^{2}}{T}\left[\sum_{k} \frac{v_{k}^{2}}{\omega_{k}}\right]\left[\sum_{p} \frac{v_{p}^{2}}{\omega_{p}}\left(q_{p}-\langle q\rangle\right)\right]\right\} \\
& \approx-0.021\left\{1+12 \cdot\left(\frac{G_{\text {freeze-out }}}{300 \mathrm{MeV}}\right)^{2}\left(\frac{\xi_{\text {freeze-out }}}{6 \mathrm{fm}}\right)^{2}\right\} \text { for } \mu_{\pi}=0 \\
& \approx-0.046\left\{1+13 \cdot\left(\frac{G_{\text {freeze-out }}}{300 \mathrm{MeV}}\right)^{2}\left(\frac{\xi_{\text {freeze-out }}}{6 \mathrm{fm}}\right)^{2}\right\} \text { for } \mu_{\pi}=60 \mathrm{MeV}
\end{aligned}
$$

where we have taken $q=p_{T}$ and $T=120 \mathrm{MeV}$. This correlation only receives contributions from nontrivial effects, and we see that near the critical point, the contribution from the interaction with the sigma field is dominant. Once again, we see that this correlation is a very interesting quantity to use to look for the critical point. As the critical point is approached, thermal contact with a heat bath whose heat capacity is diverging reduces the effect of energy conservation, as seen in (70); we now see that this reduction is overcompensated by the larger increase in the cross correlation which is induced by the direct coupling of the pions to the sigma field. The lesson is clear: although this correlation is small, it may increase in magnitude by a very large factor near the critical point.

The effects of the critical fluctuations can be detected in a number of ways. First, one can find a nonmonotonic increase in $F_{\sigma}$, the suitably normalized increase in the variance of event-by-event fluctuations of the mean transverse momentum. Second, one can find a nonmonotonic increase in $\left\langle(\Delta N)^{2}\right\rangle$. Both these effects can easily be between one and two orders of magnitude greater than the statistical errors in present data. Third, one can find a nonmonotonic increase in the magnitude of $\left\langle\Delta p_{T} \Delta N\right\rangle$. This quantity is small, and it has not yet been demonstrated that it can be measured. However, it may change at the critical point by a large factor, and is therefore worth measuring. In addition to effects on these and many other observables, it is perhaps most distinctive to measure the microscopic correlator $\left\langle\Delta n_{p} \Delta n_{k}\right\rangle$ itself. The term proportional to $1 / m_{\sigma}^{2}$ in (88) has a specific dependence on $p$ and $k$. It introduces off-diagonal correlations in $p k$ space. Like the off-diagonal anti-correlation discussed in Section 4, this makes it easy to distinguish from the Bose enhancement effect, which is diagonal in $p k$. Near the critical point, the off-diagonal anti-correlation vanishes and the off-diagonal correlation due to sigma exchange grows. Furthermore, the effect of $\sigma$ exchange is not restricted to identical pions, and should be visible as correlations between the fluctuations of $\pi^{+}$and $\pi^{-}$. The dominant diagonal term proportional to $\delta_{p k}$ in (88) will 
be absent in the correlator $\left\langle\Delta n_{p}^{+} \Delta n_{k}^{-}\right\rangle$, and the effects of $\sigma$ exchange will be the dominant contribution to this quantity near the critical point.

\section{Pions From Sigma Decay}

There is another signature of freeze-out near the critical point discussed in [1] in addition to those we have analyzed in depth above. For choices of control parameters such that freeze-out occurs at or near the critical endpoint, the excitations of the sigma field, sigma (quasi)particles, are nearly massless at freeze-out and are therefore numerous. Because the pions are massive at the critical point, these $\sigma$ 's cannot immediately decay into two pions. Instead, they persist as the temperature and density of the system further decrease. During the expansion, the in-medium $\sigma$ mass rises towards its vacuum value and eventually exceeds the two pion threshold. Thereafter, the $\sigma$ 's decay, yielding a population of pions which do not get a chance to thermalize because they are produced after freeze-out. Here, we estimate the momentum spectrum of these pions produced by delayed $\sigma$ decay. An event-by-event analysis is not required in order to see these pions. The excess multiplicity at low $p_{T}$ will appear and then disappear in the single particle inclusive distribution as control parameters are varied such that the critical point is approached and then passed.

The event-by-event fluctuations of the multiplicity of these pions reflect the fluctuations of the sigma field whence they came [1]. We estimate the resulting increase in the event-byevent fluctuations of $N$, the total pion multiplicity.

We begin with the inclusive single particle $p_{T}$-spectrum of the pions from sigma decay. We use the expression (93) for the width of the $\sigma$, but now treat $m_{\sigma}$ as time-dependent. We should also take $G$ to evolve with time. However, the dominant time-dependent effect is the opening up of the phase space for the decay as $m_{\sigma}$ increases with time and crosses the two-pion threshold. We will therefore treat $G$ as a constant. In Section 5, we estimated that in vacuum with $m_{\sigma}=600 \mathrm{MeV}$, the coupling is $G \sim 1900 \mathrm{MeV}$, whereas at the critical end point with $m_{\sigma}=0$, the coupling is reduced, perhaps by as much as a factor of six or so. In this section, we need to estimate $G$ at the time when $m_{\sigma}$ is at or just above twice the pion mass. We will use $G \sim 1000 \mathrm{MeV}$, recognizing that we may be off by as much as a factor of two.

Let us parameterize the time dependence of the sigma mass by

$$
m_{\sigma}(t)=2 m_{\pi}(1+t / \tau)
$$

where we have defined $t=0$ to be the time at which $m_{\sigma}$ has risen to $2 m_{\pi}$ and have introduced the timescale $\tau$ over which $m_{\sigma}$ increases from $2 m_{\pi}$ to $4 m_{\pi}$. We will be interested in times $0<t<\tau$, for which this linear parameterization of the time dependence is not unreasonable. Note that with this choice of notation, freeze-out occurs at a negative time, and the collision begins at an even more negative time. Substituting into (93), and working to lowest order in $t / \tau$, we find

$$
\Gamma(t) \sim \frac{3 G^{2}}{32 \pi m_{\pi}} \sqrt{2 t / \tau}=D \sqrt{t / \tau},
$$


where we have defined

$$
D=\frac{3 \sqrt{2} G^{2}}{32 \pi m_{\pi}} \sim(300 \mathrm{MeV})\left(\frac{G}{1000 \mathrm{MeV}}\right)^{2} .
$$

$N(t)$, the number of $\sigma$ 's present at time $t$, is determined by

$$
\frac{1}{N(t)} \frac{d N(t)}{d t}=-\Gamma(t)=-D \sqrt{t / \tau}
$$

and is therefore

$$
N(t)=N(0) \exp \left(-\frac{2}{3} D t^{3 / 2} \tau^{-1 / 2}\right) .
$$

We can now estimate the momentum distribution of the pions produced in the decay of the sigmas, upon making the assumption that the sigmas are at rest when they decay. This is a good approximation for two reasons. First, as the system expands after freeze-out, the sigma mass is increasing as we have discussed. This means that the kinetic energy of each sigma is decreasing. Second, during the time between freeze-out and decay, some of the sigmas which happen to be moving outwards toward the less dense region of the collision in which their mass would increase more than allowed by energy conservation will instead be reflected back inward. Each sigma which suffers such a reflection loses momentum, as the reflection occurs as if off an outward moving surface. This effect confines the sigmas to the densest region of the plasma, where their mass remains low for the longest time, and in addition reduces their momenta. We do not attempt a quantitative estimate of these two momentum-reducing effects here. Suffice to say that since at freeze-out the typical sigmas will have momenta of order the freeze-out temperature or less, we think it reasonable to approximate them as being at rest at time $t=0$ when they begin to decay.

Sigmas which decay at rest at time $t$ each yield two pions with momenta $p \sim m_{\pi} \sqrt{2 t / \tau}$, to lowest order in $t / \tau$. As a result, the number of pions with momenta $m_{\pi} \sqrt{2 t / \tau}<p<$ $m_{\pi} \sqrt{2(t+d t) / \tau}$ is $-2 d t(d N(t) / d t)$ with $N(t)$ given by (123). Upon making suitable substitutions, we find that the number of pions with momenta between $p$ and $d p$ is

$$
d N=\sqrt{2} N(0) D \tau \frac{p^{2} d p}{m_{\pi}^{3}} \exp \left(-\frac{1}{3 \sqrt{2}} D \tau \frac{p^{3}}{m_{\pi}^{3}}\right) .
$$

With the momentum distribution in hand, we determine the mean pion momentum to be

$$
\bar{p}^{\text {inc }}=2^{1 / 6} 3^{-2 / 3} \Gamma(1 / 3) m_{\pi}(D \tau)^{-1 / 3}=1.45 m_{\pi}(D \tau)^{-1 / 3} .
$$

Large $\tau$ corresponds to slow expansion and a sigma mass which consequently increases only slowly with time; large $D$ corresponds to a large coupling constant $G$. It therefore makes sense that if $D \tau$ is large, the sigmas decay before the sigma mass has increased far above threshold, and the resulting pions have small momenta. We defined $\tau$ to be the time it takes the $m_{\sigma}$ to increase from $2 m_{\pi}$ to $4 m_{\pi}$. This timescale is hard to estimate, but our result is 
not strongly dependent on $\tau$. It seems likely that $5 \mathrm{fm}<\tau<20 \mathrm{fm}$ and we therefore quote our result as

$$
\bar{p}^{\mathrm{inc}} \sim 0.58 m_{\pi}\left(\frac{1000 \mathrm{MeV}}{G}\right)^{2 / 3}\left(\frac{10 \mathrm{fm}}{\tau}\right)^{1 / 3},
$$

where we have used (121). We therefore estimate that if freeze-out occurs near the critical point, there will be a nonthermal population of pions with transverse momenta of order half the pion mass distributed according to (124).

How many such pions can we expect? That is, how large is $N(0)$ ? This is determined by the sigma mass at freeze-out. If $m_{\sigma}$ is comparable to $m_{\pi}$ at freeze-out, then there are half as many $\sigma$ 's at freeze-out as there are charged pions. Since each sigma decays into two pions, and two thirds of those pions are charged, the result is that the number of charged pions produced by sigma decays after freeze-out is $2 / 3$ of the number of charged pions produced directly by the freeze-out of the thermal pion gas. Of course, if freeze-out occurs closer to the critical point at which $m_{\sigma}$ can be as small as $(6 \mathrm{fm})^{-1}$, there would be even more sigmas. We therefore suggest that as experimenters vary the collision energy, one way they can discover the critical point is to see the appearance and then disappearance of a population of pions with $\left\langle p_{T}\right\rangle \sim m_{\pi} / 2$ which are almost as numerous as the direct pions. Yet again, it is the nonmonotonicity of this signature as a function of control parameters which makes it distinctive.

As we discussed briefly in [1], the event-by-event fluctuations in the multiplicity of these low momentum pions are also of interest. If we were able to measure the multiplicity of sigma quasiparticles at freezeout, we would find fluctuations given by

$$
\left\langle\left(\Delta N^{\sigma}\right)^{2}\right\rangle=\sum_{p}\left\langle n_{p}^{\sigma}\right\rangle\left(1+\left\langle n_{p}^{\sigma}\right\rangle\right)
$$

where the $n_{p}^{\sigma}$ 's are the sigma occupation numbers. In the present analysis, we neglect the effects of interactions among the sigmas and just take $n_{p}^{\sigma}$ as for an ideal Bose gas with small $m_{\sigma}$. We expect that this makes our prediction for the fluctuations an underestimate. Since $m_{\sigma}$ is small, the low momentum modes have large occupation number, and have fluctuations proportional to the square of their occupation number. Each sigma eventually decays into two pions, whose momenta are determined by the time at which the sigma decays, rather than by the sigma momentum at freeze-out. It is therefore not possible to make a measurement on the pions which restricts the $\sum_{p}$ in (127) to low $p_{T}$. We therefore do the entire sum, and find that the variance of the event-by-event distribution of the multiplicity of the $\sigma$-produced pions is

$$
\left\langle(\Delta N)^{2}\right\rangle=2\langle N\rangle\left(1+\overline{\left\langle n_{p}^{\sigma}\right\rangle}{ }^{\text {inc }}\right)
$$

where $N$ is the number of charged pions. The factor of two arises because every sigma which produces charged pions produces two charged pions, and was discussed in Section 3. Taking $m_{\sigma}=0$ yields $\overline{\left\langle n_{p}^{\sigma}\right\rangle}{ }^{\text {inc }} \approx 0.37$, and therefore

$$
\left\langle(\Delta N)^{2}\right\rangle \approx 2.74\langle N\rangle \text {. }
$$


We have already seen in Section 5 that the critical fluctuations of the sigma field increase the fluctuations in the multiplicity of the direct pions sufficiently that the increase in the fluctuation of the multiplicity of all the pions will be increased by about $10 \%$. We now see that in the vicinity of the critical point, there will be a further nonmonotonic rise in the fluctuations of the multiplicity of the population of pions with $\left\langle p_{T}\right\rangle \sim m_{\pi} / 2$ which are produced in sigma decay.

\section{Summary and Outlook}

In order to estimate the magnitude of the effects of critical fluctuations, one must first analyze the background, noncritical fluctuations. NA49 data from PbPb collisions at 160 $\mathrm{AGeV}$ shows that the event-by-event distribution of the mean transverse momentum is as perfect a Gaussian as the central limit theorem allows. Since a system in thermodynamic equilibrium exhibits Gaussian fluctuations, in Section 3 we give a quantitative answer to the question of how much of the observed fluctuations are thermodynamic in origin. To this end, we model the matter at freeze-out as an ideal gas of pions and resonances in thermal equilibrium, estimate the resulting fluctuations, and compare with the data.

We calculate the event-by-event fluctuations of $p_{T}$, an intensive quantity which is therefore little affected by nonthermodynamic fluctuations in the initial size of the system. We find that the resonances turn out to be of little importance - the resonance gas prediction for $\left\langle\left(\Delta p_{T}\right)^{2}\right\rangle /\left\langle p_{T}\right\rangle^{2}$ is almost indistinguishable from that of an ideal Bose gas of pions. Furthermore, we have verified quantitatively that the correlations between pions introduced by the fact that some originate in resonance decays can be neglected. We have computed the effects of Bose enhancement, and find that they increase $\left\langle\left(\Delta p_{T}\right)^{2}\right\rangle$ by only a few percent, although the precision of the data should make effects of this magnitude detectable.

The difficulty comes in the treatment of the collective flow. This hydrodynamic expansion boosts the momenta of the pions, affecting both the numerator and the denominator in $\left\langle\left(\Delta p_{T}\right)^{2}\right\rangle /\left\langle p_{T}\right\rangle^{2}$. Although we do expect that the effect cancels in the ratio to a significant extent, the "blue shift" approximation which we have used is too simple. We have shown quantitatively that the fluctuations in the flow velocity can be neglected. However, the effects of the flow itself are not sufficiently accurately treated as a uniform blue shift, and must be treated more quantitatively in the future. We find that our prediction for $\left\langle\left(\Delta p_{T}\right)^{2}\right\rangle /\left\langle p_{T}\right\rangle^{2}$ is about $90 \%$ of that which NA49 observes. This gives us further confidence that we can use thermodynamics to understand the great bulk of the observed fluctuations; improving the precision of the prediction by improving upon the blue shift approximation remains to be done.

The data are precise enough that we can do more than analyze the "bulk" of the fluctuations. We can ask, for example, about the ratio $\sqrt{F}$ of $\langle N\rangle^{1 / 2} v_{\text {ebe }}\left(p_{T}\right)$ to the variance of the inclusive single particle distribution. This ratio is insensitive to the effects of the flow velocity. For a classical ideal gas, $\sqrt{F}=1$. We estimate that Bose effects result in $\sqrt{F}=\sqrt{F_{B}} \approx 1.02$. In the data, however, $\sqrt{F}=1.002 \pm 0.002$. The Bose effects may be small, but they are ten times larger than the statistical error in the data. The Bose correlations are being compensated by some anti-correlation, and in Section 4 we find a possible explanation. 
We show that energy conservation results in an anti-correlation which is reduced by thermal contact between the direct pions and an unobserved heat bath. The anti-correlation vanishes if the heat bath has infinite heat capacity. This effect, and indeed everything about the fluctuations we analyze, can be derived from the correlator $\left\langle\Delta n_{p} \Delta n_{k}\right\rangle$ between the fluctuations of the occupation numbers of pion modes with momenta $p$ and $k$. Energy conservation implies that if $n_{p}$ fluctuates up, then $n_{k}$ is more likely to fluctuate down. The magnitude of the effect depends on the heat capacity of the "heat bath", but we estimate that it leads to $\sqrt{F}=\sqrt{F_{B} F_{T}}$ with $\sqrt{F_{T}} \approx 0.99$.

With more detailed experimental study, either now at the SPS, or soon at RHIC (STAR will study event-by-event fluctuations in $p_{T}, N$, particle ratios, etc; PHENIX and PHOBOS in $N$ only) it should be possible to disentangle the different effects we describe. Making a cut to look at only low $p_{T}$ pions should increase the effects of Bose enhancement. Bose enhancement effects are sensitive to $\mu_{\pi}$, and measuring these effects would allow one to make an experimental determination of this quantity. The anti-correlation introduced by energy conservation and thermal contact is due to terms in $\left\langle\Delta n_{p} \Delta n_{k}\right\rangle$ which are off-diagonal in $p k$. Thus, a direct measurement of $\left\langle\Delta n_{p} \Delta n_{k}\right\rangle$ would make it easy to separate this anticorrelation from other effects. The cross correlation $\left\langle\Delta N \Delta p_{T}\right\rangle$ is a very interesting observable to study because it only receives contributions from interesting effects, like Bose enhancement, thermal contact and the critical fluctuations discussed in Section 5. We hope that the combination of the theoretical tools we have provided and the present NA49 data provide a solid foundation for the future study of the thermodynamics of the hadronic matter present at freeze-out in heavy ion collisions.

We also consider fluctuations in multiplicity $N$, an extensive observable. These are not affected by the boost which the pion momenta receive from the collective flow, and this makes them easier to calculate than the fluctuations in $p_{T}$. However, multiplicity fluctuations are contaminated experimentally by fluctuations in the initial state, for example due to the distribution of impact parameters. This experimental contamination can be reduced by making a tight enough centrality cut using a zero degree calorimeter. We compare the multiplicity fluctuations of the $5 \%$ most central events in the NA49 data to those we predict from a resonance gas, and find evidence that about $75 \%$ of the observed fluctuation is indeed thermodynamic in origin. We find that resonances play a significant role in this comparison, increasing the magnitude of thermodynamic fluctuations of the pion multiplicity and bringing it closer to the data.

With the foundations established, we then describe how the fluctuations we analyze will change if control parameters are varied in such a way that the baryon chemical potential at freeze-out, $\mu_{\mathrm{f}}$, moves toward and then past the critical point in the QCD phase diagram at which a line of first order transitions ends at a second order endpoint. We provide quantitative estimates of the magnitude of the change in the observables we have analyzed which can be expected near this point. The agreement between the noncritical thermodynamic fluctuations in $p_{T}$ which we analyze in Section 3 and NA49 data make it unlikely that central $\mathrm{PbPb}$ collisions at $160 \mathrm{AGeV}$ freeze out near the critical point. Estimates we have made in a previous paper suggest that the critical point is located at a baryon chemical potential $\mu$ such that it will be found at an energy between $160 \mathrm{AGeV}$ and AGS energies. This makes it a prime target for detailed study at the CERN SPS by comparing data taken at $40 \mathrm{AGeV}$, 
$160 \mathrm{AGeV}$, and in between. If the critical point is located at such a low $\mu$ that the maximum SPS energy is insufficient to reach it, it would then be in a regime accessible to study by the RHIC experiments. We want to stress that we are more confident in our ability to describe the properties of the critical point and thus to predict how to find it than we are in our ability to predict where it is.

The critical fluctuations near the endpoint affect the event-by-event fluctuations which we analyze in two different ways. First, all effects of energy conservation should be greatly reduced by thermal contact as the critical fluctuations in the sigma field cause the heat capacity to grow. Second, these critical fluctuations have direct effects on the fluctuations of the pions through the $G \sigma \pi \pi$ coupling. We analyze the most singular effects of this coupling, which are due to the zero momentum mode of the sigma field. It is possible to analyze subleading corrections using a diagrammatic approach, but we leave this to the future.

In the chiral limit, the critical point becomes a tricritical point at which $G$ vanishes. We estimate the vacuum value of $G$ and use scaling laws valid near a tricritical point to estimate $G$ at the critical point. We then estimate the increase in the fluctuations of $N$ and $p_{T}$ distributions which we expect in heavy ion collisions which freeze out near the critical point. Finite size and finite time effects prevent $\left\langle(\Delta N)^{2}\right\rangle /\langle N\rangle$ and $\left\langle\left(\Delta p_{T}\right)^{2}\right\rangle\langle N\rangle$ from diverging, as they would in an infinite system. We estimate that $\left\langle(\Delta N)^{2}\right\rangle /\langle N\rangle$ can grow by more than $10 \%$. The ratio $\sqrt{F}$ which describes the $p_{T}$-fluctuations becomes $\sqrt{F_{B} F_{T} F_{\sigma}}$ with $\sqrt{F_{\sigma}}$ about 1.1. This effect is not large but is still predicted to be 50 times larger than the statistical error in the present NA49 measurement of $\sqrt{F}=1.002 \pm 0.002$. We quantify the uncertainty in our estimates in terms of the sigma correlation length $\xi$ and the coupling $G$ at freezeout; measurement of the enhanced fluctuations of $N$ and $p_{T}$ would allow one to estimate $G \xi$.

We want to emphasize that the ratio $\sqrt{F}$ is not the only observable which can be used to detect the proximity of the critical point, and indeed is not the most sensitive observable available. We have focussed on $\sqrt{F}$ because it is simple to define, and because NA49 has published data to which we can compare our predictions. However, the specific form of the singularity in $\left\langle\Delta n_{p} \Delta n_{k}\right\rangle$ which we find in Eq. (88) tells us how to construct observables which are more sensitive to the critical fluctuations. One possibility is to consider observables which are sensitive to the off-diagonal part of $\left\langle\Delta n_{p} \Delta n_{k}\right\rangle$, because the noncritical off-diagonal anticorrelation in $\left\langle\Delta n_{p} \Delta n_{k}\right\rangle$ should be replaced by a much larger off-diagonal correlation near the critical point. A second possibility is an analysis of the cross correlation $\left\langle\Delta N \Delta p_{T}\right\rangle$. Because this cross correlation is dominated by interesting effects, we have seen that it can increase by an order of magnitude at the critical point. A third possibility is to construct a ratio like $\sqrt{F}$, but using only soft pions, with $p_{T}$ less than a specified cutoff. The effects of the critical fluctuations are largest on the softest pions, and they are therefore masked in $\sqrt{F}$ which receives significant contribution from harder particles. Whereas we have found that the critical fluctuations change $\sqrt{F}$ at the $10 \%$ level, their effect on a "soft $\sqrt{F}$ " can easily be at the factor of two level.

Although the sigma quasiparticles themselves cannot be reconstructed, their presence can be detected even more directly than via their influence on the pions at freeze-out. If freezeout occurs near the critical point, some time after freeze-out the sigma mass rises above the two pion threshold, and the sigmas decay quickly. Since these pions do not rethermalize, the resulting excess in the low- $p_{T}$ region of the pion momentum spectrum should be observable. 
The mean $p_{T}$ of these pions is about $m_{\pi} / 2$, and they are almost as numerous as the direct pions. The event-by-event fluctuations in the multiplicity of these soft pions would be even larger than those of the rest of the pions near the critical point.

In summary, our understanding of the thermodynamics of QCD will be greatly enhanced by the detailed study of event-by-event fluctuations in heavy ion collisions. We have estimated the influence of a number of different physical effects, some special to the vicinity of the critical point but many not, on the fundamental correlator $\left\langle\Delta n_{p} \Delta n_{k}\right\rangle$. This is itself measurable, but we have in addition used it to make predictions for the fluctuations of observables which have been measured at present, like $\left\langle\left(\Delta p_{T}\right)^{2}\right\rangle$ and $\left\langle(\Delta N)^{2}\right\rangle$ and also for the cross correlation $\left\langle\Delta N \Delta p_{T}\right\rangle$. The predictions of a simple resonance gas model, which does not include critical fluctuations, is to this point in broad agreement with the data. More detailed study, for example with varying cuts in addition to new observables, will help to further constrain the nonthermodynamic fluctuations, which are clearly small, and better understand the different thermodynamic effects. The signatures we analyze allow experiments to map out distinctive features of the QCD phase diagram. The striking example which we have considered in detail is the effect of a second order critical end point. The nonmonotonic appearance and then disappearance of any one of the signatures of the critical fluctuations which we have described would be strong evidence for the critical point. Furthermore, if a nonmonotonic variation is seen in several of these observables, then the maxima in all the signatures must occur simultaneously, at the same value of the control parameters. Simultaneous detection of the effects of the critical fluctuations on different observables would turn strong evidence into an unambiguous discovery.

\section{Acknowledgements}

We are grateful to G. Roland for providing us with preliminary NA49 data. We acknowledge helpful conversations with M. Creutz, U. Heinz, M. Gaździcki, V. Koch, St. Mrówczyński, G. Roland and T. Trainor.

The work of MS is supported by NSF grant PHY97-22101. The work of KR is supported in part by a DOE Outstanding Junior Investigator Award, by the Alfred P. Sloan Foundation, and by the DOE under cooperative research agreement DE-FC02-94ER40818. The work of ES is supported in part by DOE grant DE-FG02-88ER40388.

\section{Note added in proof}

As we have stressed in Section 3, the fluctuations in an extensive quantity such as the observed multiplicity are unlike fluctuations in intensive quantities in that they receive significant contributions from both (i) thermodynamic fluctuations at freeze-out and (ii) nonthermodynamic fluctuations during the initial stage of the collision. Our approach has been to use a comparison between the data and thermodynamic predictions to constrain the magnitude of non-thermodynamic fluctuations.

After this paper was submitted, Ref. [33] appeared. These authors have attempted a theoretical treatment of those non-thermodynamic fluctuations which are purely geometrical in that they can be attributed to the distribution of impact parameters. Further analysis is 
presented in Ref. [34. These authors include in addition the effects of fluctuations in the NN cross-section [17], which they find to be small, and also fluctuations in the number of punchthrough spectators and effects due to the diffuse edges of the incident nuclei, both of which are significant. Combining all contributions to the multiplicity fluctuations, thermodynamic and nonthermodynamic, yields fluctuations which, with no new free parameters, reproduce the magnitude of the observed multiplicity fluctuations to within a few percent accuracy 34 .

\section{A Finiteness of Multiplicity}

Throughout the body of the paper, we use event-by-event and inclusive averages defined probabilistically. If we were interested in an infinite ensemble in which each member of the ensemble was in the thermodynamic limit, no translation would be required. However, when we want to compare the relations involving quantities which are defined probabilistically to those measured in an experiment, as in any application of probability theory we must have estimators for these quantities which can be constructed from finite samples. In this Appendix, we discuss the effects due to finite sample size.

The typical size of these effects is one over a power of the sample size. The total number, $C$, of events can be easily made very large (say, $10^{6}$ ), so that $1 / C$, and even $1 / \sqrt{C}$ is much smaller than the physical effects we consider, (such as Bose enhancement, for example) which are of the order of a few percent or more. However, the number of pions in an event, $N$, is limited by the size of the colliding system and the experimental acceptance of a detector, and is typically of the order of a few hundred. This can introduce corrections of the order of a fraction of a percent. Of course, these effects are negligible when compared to the bulk of the fluctuations, which we analyze in Section 3. They are also smaller than the effects we discuss in Sections 4 and 5, where we are interested in signatures which rise and fall by of order $10 \%$ near the critical point. However, we have seen in Section 3 that the statistical errors in the present data are small enough that one can compare quantities like $\langle N\rangle v_{\text {ebe }}^{2}$ and

$v_{\text {inc }}^{2}$ to a precision of less than a percent. At this level, we must understand how to deal with the $1 / N$ corrections.

Let us consider a sample of values of some one-particle observable $q$. This sample is broken into $C$ subsets, i.e., events, with $N_{a}$ values per event. We use the notation: $q_{i}^{a}$, where $a=1 \ldots C$ and $i=1 \ldots N_{a}$. (For example, $q_{i}^{a}$ may be the momentum of the $i$ 'th pion in the $a^{\prime}$ th event.) The numbers $q_{i}^{a}$ are distributed according to some (joint) probability distribution. We assume that the expectation value is the same for all $q_{i}^{a}: M\left[q_{i}^{a}\right]=m$.

In Section 2 we pointed out that the inclusive mean $\bar{q}^{\text {inc }}$ is the same as the event-by-event average $\langle q\rangle$. Both quantities are defined in the infinite $C$ and $N$ limit. Let us now try to estimate these two quantities, using our finite sample $q_{i}^{a}$. The natural estimate for $\bar{q}^{\text {inc }}$ is the following:

$$
\bar{q}_{\mathrm{est}}^{\mathrm{inc}}=\frac{\sum_{a=1}^{C} \sum_{i=1}^{N_{a}} q_{i}^{a}}{\sum_{a=1}^{C} N_{a}}=\frac{1}{C\langle N\rangle} \sum_{a=1}^{C} \sum_{i=1}^{N_{a}} q_{i}^{a}
$$


where we have introduced (somewhat inconsistently, but suggestively):

$$
\langle N\rangle=\frac{1}{C} \sum_{a=1}^{C} N_{a}
$$

which is (an estimate for) the mean multiplicity in an event. The total number of $q$ 's in the sample is $C\langle N\rangle$. The property of the estimate (130) is that its expectation value is equal to $m$ :

$$
M\left[\bar{q}_{\mathrm{est}}^{\mathrm{inc}}\right]=m,
$$

for any $N$ or $C$. The standard deviation of this quantity is $O(1 / \sqrt{C\langle N\rangle})$, so the estimate becomes perfect in the infinite $C, N$ limit. Now let us estimate the event-by-event mean of q. An estimate which appears natural is:

$$
\langle q\rangle_{\mathrm{est} 0}=\frac{1}{C} \sum_{a=1}^{C}\left(\frac{1}{N_{a}} \sum_{i=1}^{N_{a}} q_{i}^{a}\right) .
$$

The expectation value of this estimate is:

$$
M\left[\langle q\rangle_{\mathrm{est} 0}\right]=m,
$$

and this estimate also becomes perfect as $C, N$ go to infinity. However,

$$
\langle q\rangle_{\mathrm{est} 0} \neq \bar{q}_{\mathrm{est}}^{\mathrm{inc}} \text {. }
$$

One can show that the difference between the two is on the order of $\left\langle(\Delta N)^{2}\right\rangle / N^{2} \sim 1 / N$. It is obvious how to improve the estimate (133) to make the relationship $\langle q\rangle=\bar{q}^{\text {inc }}$ hold exactly for finite $N$. Writing:

$$
\bar{q}_{\mathrm{est}}^{\mathrm{inc}}=\frac{1}{C} \sum_{a=1}^{C} \frac{N_{a}}{\langle N\rangle}\left(\frac{1}{N_{a}} \sum_{i=1}^{N_{a}} q_{i}^{a}\right) \stackrel{\text { def }}{=}\langle q\rangle_{\mathrm{est}},
$$

we can interpret this definition of the estimate $\langle q\rangle_{\text {est }}$ as a result of averaging over events with a weight proportional to the multiplicity in this event, $N_{a}$. It is also clear intuitively that such a procedure is more natural than taking all events with equal weight as is done in (133). What is important is that, by construction, this procedure rids us of any $1 / N$ correction to the equality between $\langle q\rangle_{\text {est }}$ and $\bar{q}_{\text {est }}^{\text {inc }}$.

Let us now consider estimating variances of $q$. The natural estimate for the inclusive square variance is:

$$
v_{\text {inc }}^{2}(q)_{\mathrm{est}}=\frac{1}{C\langle N\rangle-1} \sum_{a=1}^{C} \sum_{i=1}^{N_{a}}\left(q_{i}^{a}-\bar{q}_{\mathrm{est}}^{\mathrm{inc}}\right)^{2} .
$$

Assuming that the variables $q_{i}^{a}$ are uncorrelated and their dispersions are equal, i.e.:

$$
M\left[q_{i}^{a} q_{j}^{b}\right]=m^{2}+\sigma^{2} \delta_{i j} \delta^{a b}
$$


one can show that

$$
M\left[v_{\text {inc }}^{2}(q)_{\mathrm{est}}\right]=\sigma^{2},
$$

for any $C$, or $N$. This is the property which we require of this estimate.

Next, we consider estimating the event-by-event variance. One seemingly natural candidate is:

$$
v_{\text {ebe }}^{2}(q)_{\text {est } 0}=\frac{1}{C-1} \sum_{a=1}^{C}\left(\frac{1}{N_{a}} \sum_{i=1}^{N_{a}} q_{i}^{a}-\langle q\rangle_{\text {est }}\right)^{2} .
$$

This is the procedure used by NA49 to calculate $v_{\text {ebe }}\left(p_{T}\right)$ from their data, leading to the result shown in Table 2. Let us calculate the expectation value of this quantity, assuming that all $q$ 's are independent as in (138). We find:

$$
M\left[v_{\mathrm{ebe}}^{2}(q)_{\mathrm{est} 0}\right]=\sigma^{2} \frac{C}{C-1}\left(\left\langle\frac{1}{N}\right\rangle-\frac{1}{C\langle N\rangle}\right) \approx \sigma^{2}\left\langle\frac{1}{N}\right\rangle,
$$

where in the last approximate equality we neglected terms of relative size $\mathcal{O}(1 / C)$. In the case of completely uncorrelated $q$ we expect the following relation to hold between the $v_{\text {ebe }}^{2}(q)$ and $v_{\text {inc }}^{2}(q)$ :

$$
\langle N\rangle v_{\text {ebe }}^{2}(q)=v_{\text {inc }}^{2}(q) .
$$

This equality cannot and should not be satisfied for an arbitrary sample (unlike the equality $\left.\langle q\rangle=\bar{q}^{\text {inc }}\right)$, but we want it to be satisfied on the level of expectation values:

$$
\langle N\rangle M\left[v_{\mathrm{ebe}}^{2}(q)_{\mathrm{est} 0}\right]=M\left[v_{\mathrm{inc}}^{2}(q)_{\mathrm{est}}\right] .
$$

We see that for the estimate (140) the difference between the l.h.s. and the r.h.s of eq.(143) is

$$
\langle N\rangle M\left[v_{\mathrm{ebe}}^{2}(q)_{\mathrm{est} 0}\right]-M\left[v_{\mathrm{inc}}^{2}(q)_{\mathrm{est}}\right] \approx \sigma\left(\langle N\rangle\left\langle\frac{1}{N}\right\rangle-1\right) \approx \sigma^{2} \frac{\left\langle(\Delta N)^{2}\right\rangle}{\langle N\rangle^{2}}
$$

where we have neglected the $\mathcal{O}(1 / C)$ corrections and the corrections which are higher order in $1 / N$. The difference (144) is a $1 / N$ effect, of course, but with $N \sim 300$ it could easily reach a fraction of a percent. Note that we are not talking here about statistical fluctuations around the mean values which make the two estimates deviate from sample to sample. Such effects are of the order $1 / \sqrt{C}$ and can easily be made negligible with sufficient statistics. Equation (144), on the other hand, reflects a systematic discrepancy between the expectation values of the estimates, which would only go away if $N$, the number of particles in one event, were infinite.

Our task now is to give an estimator for $v_{\text {ebe }}^{2}(q)$ which satisfies (143) with no errors of order $1 / N$ in the case when the $q_{i}^{a}$ are uncorrelated. The lesson we learned from the estimator for $\langle q\rangle$ suggests that we take each event with the weight $N_{a} /\langle N\rangle$. This gives:

$$
v_{\text {ebe }}^{2}(q)_{\text {est }}=\frac{1}{C-1} \sum_{a=1}^{C} \frac{N_{a}}{\langle N\rangle}\left(\frac{1}{N_{a}} \sum_{i=1}^{N_{a}} q_{i}^{a}-\langle q\rangle_{\text {est }}\right)^{2} .
$$


Calculating the expectation value we find that

$$
M\left[v_{\text {ebe }}^{2}(q)_{\text {est }}\right]=\frac{\sigma^{2}}{\langle N\rangle}
$$

exactly! This means that the estimate (145) satisfies our criterion (143) exactly, to all orders in $1 / N$ and $1 / C$. This is the estimate for $v_{\text {ebe }}^{2}(q)$ that should be used to analyze experimental data. It introduces no $1 / N$ or $1 / C$ errors in the statement that the ratio $F=1$ in the absence of correlation or interaction between the pions.

We can avoid having to apply the formula (145) to the original experimental data set in order to recalculate the $v_{\text {ebe }}\left(p_{T}\right)$ given in Table 2, which used the estimate (140). Using our

analysis, we can instead just use the fact that (the expectation values of) the two estimates are related by (see (144)):

$$
v_{\text {ebe }}(q)_{\text {est }}=v_{\text {ebe }}(q)_{\text {est } 0}\left(1-\frac{1}{2} \frac{\left\langle(\Delta N)^{2}\right\rangle}{\langle N\rangle^{2}}\right),
$$

up to corrections which are higher order in $1 / N$ (and corrections of order $1 / \sqrt{C}$ ). We use this relation to convert from one estimate to another in Eq. (42) of Section 3.4.

To finish the discussion of the $1 / N$ effects we also point out that yet another estimate for $v_{\text {ebe }}(q)$ is used implicitly in the definition of $\Phi_{p_{T}}$ in [8, 21]:

$$
\Phi_{p_{T}}=\langle N\rangle^{1 / 2} v_{\text {ebe }}\left(p_{T}\right)_{\text {est } \Phi}-v_{\text {inc }}\left(p_{T}\right) .
$$

The definition of $v_{\text {ebe }}\left(p_{T}\right)_{\text {est } \Phi}$ corresponds, in the language that we use here, to giving each event a weight $N_{a}^{2} /\langle N\rangle^{2}$ (and using $C$ instead of $C-1$ ):

$$
v_{\text {ebe }}^{2}(q)_{\text {est } \Phi}=\frac{1}{C} \sum_{a=1}^{C} \frac{N_{a}^{2}}{\langle N\rangle^{2}}\left(\frac{1}{N_{a}} \sum_{i=1}^{N_{a}} q_{i}^{a}-\langle q\rangle_{\text {est }}\right)^{2} .
$$

Calculating the expectation value of this estimate, one finds:

$$
M\left[v_{\mathrm{ebe}}^{2}(q)_{\mathrm{est} \Phi}\right]=\sigma^{2}\left(\frac{1}{\langle N\rangle}-\frac{\left\langle N^{2}\right\rangle}{C\langle N\rangle^{3}}\right) \approx \frac{\sigma^{2}}{\langle N\rangle},
$$

where we neglected $\mathcal{O}(1 / C)$ corrections in the last step. We see that in the $C \rightarrow \infty$ limit this estimate does not suffer from $1 / N$ corrections as far as the relation (143) is concerned, and does not differ from the estimate (145). However, it does introduce small $1 / C$ corrections, while the estimate (145) satisfies (143) exactly.

\section{References}

[1] M. Stephanov, K. Rajagopal, E. Shuryak, Phys. Rev. Lett. 81 (1998) 4816.

[2] J. Berges and K. Rajagopal, Nucl. Phys. B538 (1999) 215.

[3] M. A. Halasz, A. D. Jackson, R. E. Shrock, M. A. Stephanov and J. J. M. Verbaarschot, Phys. Rev. D58 (1998) 096007. 
[4] The fact that larger systems freezeout later has been established experimentally by seeing the $A$-dependence of the freeze-out temperature via analyses of flow[20], Coulomb effects (H. W. Barz, J. P. Bondorf, J. J. Gaardhoje and H. Heiselberg, Phys. Rev. C57 (1998) 2536), and pion interferometry (U. Heinz, Proceedings of Quark Matter '97, nucl-th/9801050).

[5] For a review see, for example, W. Trautmann, nucl-ex/9611002.

[6] M. L. Gilkes et al., Phys. Rev. Lett. 73 (1994) 1590; J. Pochodzalla et al., Phys. Rev. Lett. 75 (1995) 1040.

[7] NA49 Collaboration, paper to be submitted. G. Roland, private communication. Talk by G. Roland at Trento Workshop on Event-by-Event Physics in Relativistic Heavy Ion Collisions, October 1998. See also G. Roland for the NA49 Collaboration, Proceedings of Quark Matter '97, Nucl. Phys. A638 (1998) 91c and talk by G. Roland, Proceedings of the Hirschegg Workshop on QCD Phase Transitions, January 1997, p. 309.

[8] M. Gaździcki, St. Mrówczyński, Z. Phys. C26 (1992) 127;

[9] M. Gaździcki, A. Leonidov and G. Roland, Eur. Phys. J. C6 (1999) 365.

[10] L. Stodolsky, Phys. Rev. Lett. 75 (1995) 1044.

[11] E. V. Shuryak, Phys. Lett. B423 (1998) 9.

[12] St. Mrówczyński, Phys. Lett. B430 (1998) 9.

[13] M. Gaździcki, nucl-th/9712050.

[14] St. Mrówczyński, nucl-th/9901078.

[15] WA98 Collaboration (M. M. Aggarwal et al.) Phys. Lett. B420 (1998) 169.

[16] See, for example, H. Heiselberg and A. D. Jackson, nucl-th/9809013 and I. N. Mishustin, hep-ph/9811307.

[17] G. Baym, G. Friedman and I. Sarcevic, Phys. Lett. 219B (1989) 205; H. Heiselberg, G.A. Baym, B. Blättel, L.L. Frankfurt and M. Strikman, Phys. Rev. Lett 67 (1991) 2946; B. Blättel, G.A. Baym, L.L. Frankfurt, H. Heiselberg and M. Strikman, Nucl. Phys. A544 (1992) 479c; G. Baym, B. Blättel, L.L. Frankfurt, H. Heiselberg and M. Strikman, Phys. Rev. C52 (1995) 1604.

[18] L. D. Landau and E. M. Lifshitz, Statistical Physics, Part 1 (Pergamon, 1980).

[19] E. Shuryak, Phys. Lett. B207 (1988) 345, Phys. Rev. D42 (1990) 1764. P. Gerber, H. Leutwyler and J. L. Goity, Phys. Lett. B246 (1990) 513. H. Bebie, P.Gerber, J. L. Goity, H. Leutwyler, Nucl. Phys. B378 (1992) 95.

[20] C. M. Hung and E. Shuryak, Phys. Rev. C57 (1998) 1891. 
[21] St. Mrówczyński, Phys. Lett. B439 (1998) 6.

[22] E.V.Shuryak, Sov. J. Nucl. Phys. 16 (1973) 220

[23] P. Braun-Munzinger and J. Stachel, Nucl. Phys. A606 (1996) 320.

[24] See, for example, S. Gottlieb et al., Phys. Rev. D55 (1997) 6852.

[25] For a review, see U. Heinz and B. Jacak, nucl-th/9902020, to appear in Ann. Rev. Nucl. Part. Sci.

[26] E. Schnedermann, J. Sollfrank, U. Heinz, Phys. Rev. C48 (1993) 2462.

[27] G. Bhanot, M. Creutz and H. Neuberger, Nucl. Phys. B235 (1984) 417.

[28] T. Trainor, talk at 15th Nuclear Dynamics Workshop, Park City, Jan. 1999.

[29] M. Gell-Mann and M. Lévy, Nuovo Cimento 16 (1960) 705.

[30] A. Barducci, R. Casalbuoni, G. Pettini and R. Gatto, Phys. Rev. D49 (1994) 426.

[31] K. Rajagopal, in Quark-Gluon Plasma 2, ed. R. C. Hwa, (World Scientific, 1995) 484.

[32] P. C. Hohenberg and B. I. Halperin, Rev. Mod. Phys. 49 (1977) 435.

[33] G. Baym and H. Heiselberg, nucl-th/9905022.

[34] G.Danilov and E. V. Shuryak, Stony Brook preprint NTG-99-27, in preparation. 